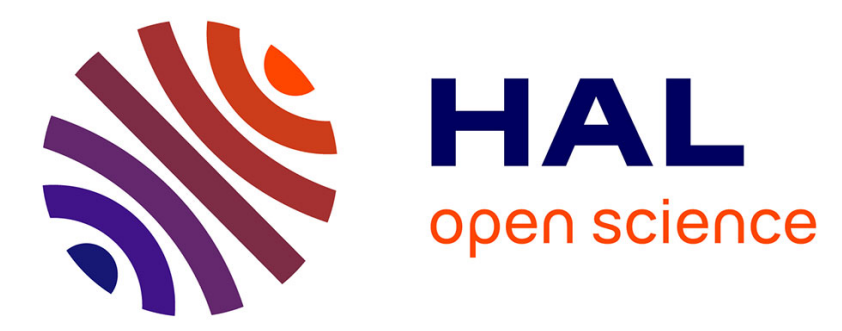

\title{
No-Reference Image Quality Assessment and Blind Deblurring with Sharpness Metrics Exploiting Fourier Phase Information
}

Arthur Leclaire, Lionel Moisan

\section{- To cite this version:}

Arthur Leclaire, Lionel Moisan. No-Reference Image Quality Assessment and Blind Deblurring with Sharpness Metrics Exploiting Fourier Phase Information. Journal of Mathematical Imaging and Vision, 2015, 52 (1), pp.145-172. 10.1007/s10851-015-0560-5 . hal-00933628v3

\section{HAL Id: hal-00933628 \\ https://hal.science/hal-00933628v3}

Submitted on 13 Oct 2015

HAL is a multi-disciplinary open access archive for the deposit and dissemination of scientific research documents, whether they are published or not. The documents may come from teaching and research institutions in France or abroad, or from public or private research centers.
L'archive ouverte pluridisciplinaire HAL, est destinée au dépôt et à la diffusion de documents scientifiques de niveau recherche, publiés ou non, émanant des établissements d'enseignement et de recherche français ou étrangers, des laboratoires publics ou privés. 


\title{
No-reference image quality assessment and blind deblurring with sharpness metrics exploiting Fourier phase information
}

\author{
Arthur Leclaire • Lionel Moisan
}

\begin{abstract}
It has been known for more than 30 years that most of the geometric content of a digital image is encoded in the phase of its Fourier transform. This has led to several works that exploit the global (Fourier) or local (Wavelet) phase information of an image to achieve quality assessment, edge detection, and, more recently, blind deblurring. We here propose a deeper insight into three recent sharpness metrics (Global Phase Coherence, Sharpness Index and a simplified version of it), which all measure in a probabilistic sense the surprisingly small total variation of an image compared to that of certain associated random-phase fields. We exhibit several theoretical connections between these indices, and study their behavior on a general class of stationary random fields. We also use experiments to highlight the behavior of these metrics with respect to blur, noise and deconvolution artifacts (ringing). Finally, we propose an application to isotropic blind deblurring and illustrate its efficiency on several examples.
\end{abstract}

Keywords phase coherence, total variation, Fourier transform, random phase noise, no-reference image quality assessment, image sharpness, blind deblurring, oracle deconvolution filter

\section{Introduction}

In several mathematical fields, the Fourier transform has shown to be a useful tool of analysis and processing. Most linear filtering operations, which are constantly used in signal and image processing, are expressed in spectral domain as simple multiplications. However, if the modulus part of the Fourier coefficients of an image is quite well understood (in particular because of

Université Paris Descartes, Sorbonne Paris Cité, France MAP5, CNRS UMR 8145

\{arthur.leclaire, lionel.moisan\}@parisdescartes.fr the link that exists between the regularity of a signal and the decrease rate of its Fourier coefficients at infinity), the argument part (the phase information) is much more difficult to apprehend. In 1981, Oppenheim and Lim [30] showed that the loss of the phase information of an image entails the destruction of the image geometry. This suggests that the precision of the image geometry (and thus, in some sense, the image quality) could be assessed through the coherence of the Fourier phase information.

Quality indices divide into three categories : fullreference, reduced-reference, and no-reference, depending on whether a supposedly ideal version of the image is assumed to be fully or partially known. As concerns the no-reference case (which is the one we are interested in), the introduction of Chapter 4 of [38] points out the difficulty to design generic image quality measures, concluding (in 2006) that "the design of application-specific no-reference quality assessment systems appears to be much more approachable than the general, assumption-free no-reference image quality assessment problem." Nevertheless, several interesting noreference quality measures have been proposed in the literature (see the recent review [7]). Some of them try to assess the quality through the direct analysis of edges [26] or through the gradient singular values [40]. Others use a perceptual analysis of certain image features, like in [13]. The concept of local phase coherence, originally introduced and developed in $[29,19,20]$ for edge detection purposes, was later linked to the perception of blur by Wang and Simoncelli [39], which ultimately led to the definition of a no-reference image quality index [17]. Closer to our work lies the index [37] which combines some spectral and spatial characteristics.

In 2008, a notion of global phase coherence was proposed [3], and related to image sharpness. The idea was 
to use a kind of a contrario framework ${ }^{1}$ [10] to quantize how much the regularity of the image (more precisely, its total variation) was affected by the destruction of the phase information. As a sharp (or noise-free) image is much more sensitive to phase degradations than a blurry (or noisy) image, such a characterization of phase coherence is directly related to image quality. This approach led to the definition of three phase coherence measures, namely the Global Phase Coherence [3], the Sharpness Index [4], and the index $S$ [22]. The present paper gives a more detailed and merged discussion about these global phase coherence indices. Starting from their construction in Section 2, we establish some of their mathematical properties in Section 3. Section 4 discusses several practical aspects of these indices, including their validation as no-reference quality measures, and finally Section 5 describes a way to use these indices to address the blind deblurring problem.

\section{Three Phase Coherence Indices}

This Section presents the detailed construction of the phase coherence indices introduced in $[3,4,22]$.

\subsection{Main notations}

In all the following, we consider discrete $M \times N$ images defined on the rectangular domain

$\Omega=\mathbb{Z}^{2} \cap\left(\left[-\frac{M}{2}, \frac{M}{2}\right) \times\left[-\frac{N}{2}, \frac{N}{2}\right)\right)$

A gray-level image is thus a map $u: \Omega \rightarrow \mathbb{R}$, the real number $u(\mathbf{x})$ referring to the gray level at pixel $\mathbf{x}$. The $\Omega$-periodization of $u$ is the image $\dot{u}: \mathbb{Z}^{2} \rightarrow \mathbb{R}$ defined by

$\forall(k, l) \in \mathbb{Z}^{2}, \forall(x, y) \in \Omega, \dot{u}(x+k M, y+l N)=u(x, y)$.

In the sequel, we will use a gradient scheme computed with periodic boundary conditions,

$\nabla u(x, y)=\left(\begin{array}{l}\partial_{x} \dot{u}(x, y) \\ \partial_{y} \dot{u}(x, y)\end{array}\right)=\left(\begin{array}{l}\dot{u}(x+1, y)-\dot{u}(x, y) \\ \dot{u}(x, y+1)-\dot{u}(x, y)\end{array}\right)$,

and the corresponding (periodic) Total Variation (TV) of $u$

$\mathrm{TV}(u)=\sum_{\mathbf{x} \in \Omega}\left|\partial_{x} \dot{u}(\mathbf{x})\right|+\left|\partial_{y} \dot{u}(\mathbf{x})\right|$,

1 The principle of a contrario methods is to detect structures as the cause of measurements that could hardly be observed in random data. which measures in some sense how much the function $\dot{u}$ oscillates. Precisely, $T V(u)$ is the $l^{1}$-norm of the gradient of $u$, and thus it assigns small values (relatively to the $l^{2}$-norm) to images whose gradient is sparse (in particular cartoon images). Algorithms based on TV minimization have been used for a long time to address image processing tasks, for example, denoising [32,6].

The discrete Fourier transform (DFT) of $u$ is the $\Omega$-periodic complex function $\hat{u}: \mathbb{Z}^{2} \rightarrow \mathbb{C}$ defined by

$\forall \boldsymbol{\xi} \in \mathbb{Z}^{2}, \quad \hat{u}(\boldsymbol{\xi})=\sum_{\mathbf{x} \in \Omega} u(\mathbf{x}) e^{-i\langle\boldsymbol{\xi}, \mathbf{x}\rangle}$,

where $\langle\boldsymbol{\xi}, \mathbf{x}\rangle=2 \pi\left(\frac{x_{1} \xi_{1}}{M}+\frac{x_{2} \xi_{2}}{N}\right)$ for $\boldsymbol{\xi}=\left(\xi_{1}, \xi_{2}\right)$ and $\mathbf{x}=\left(x_{1}, x_{2}\right)$. The function $|\hat{u}|$ will be called modulus of $u$. A phase function for $u$ is any function $\varphi: \mathbb{Z}^{2} \rightarrow \mathbb{R}$ such that for all $\boldsymbol{\xi} \in \mathbb{Z}^{2}, \hat{u}(\boldsymbol{\xi})=|\hat{u}(\boldsymbol{\xi})| e^{i \varphi(\boldsymbol{\xi})}$. If $\hat{u}(\boldsymbol{\xi}) \neq 0$, the phase coefficient $\varphi(\boldsymbol{\xi})$ is uniquely defined modulo $2 \pi$ while any arbitrary value can be chosen if $\hat{u}(\boldsymbol{\xi})=0$.

Among useful properties of the DFT, we have the reconstruction formula

$\forall \mathbf{x} \in \mathbb{Z}^{2}, \quad \dot{u}(\mathbf{x})=\frac{1}{M N} \sum_{\boldsymbol{\xi} \in \Omega} \hat{u}(\boldsymbol{\xi}) e^{i\langle\boldsymbol{\xi}, \mathbf{x}\rangle}$.

Also, the circular convolution of two images $u$ and $v$ defined by

$\forall \mathbf{x} \in \Omega, \quad u * v(\mathbf{x})=\sum_{\mathbf{y} \in \Omega} \dot{u}(\mathbf{x}-\mathbf{y}) v(\mathbf{y})$

satisfies $\widehat{u * v}=\hat{u} \hat{v}$.

We shall also need the (non-necessarily integer) $\mathrm{Ny}$ quist frequencies denoted by $\boldsymbol{\eta}_{x}=\left(-\frac{M}{2}, 0\right), \boldsymbol{\eta}_{y}=$ $\left(0,-\frac{N}{2}\right), \boldsymbol{\eta}_{x y}=\left(-\frac{M}{2},-\frac{N}{2}\right)$. When integer, these are (with zero) the only points $\boldsymbol{\xi} \in \Omega$ which are equal to $-\boldsymbol{\xi}$ modulo $(M, N)$. This allows us to define precisely the notion of random phase with

Definition 1 ([16]) A (uniform) random phase function is a function $\psi: \Omega \rightarrow \mathbb{R}$ such that

- $\psi$ is odd

- $\forall \boldsymbol{\xi} \in \Omega \backslash\left\{0, \boldsymbol{\eta}_{x}, \boldsymbol{\eta}_{y}, \boldsymbol{\eta}_{x y}\right\}, \psi(\boldsymbol{\xi})$ is uniform on $[-\pi, \pi)$

- $\forall \boldsymbol{\xi} \in\left\{0, \boldsymbol{\eta}_{x}, \boldsymbol{\eta}_{y}, \boldsymbol{\eta}_{x y}\right\}, \psi(\boldsymbol{\xi})$ is uniform on $\{0, \pi\}$

- for every subset $S$ of $\Omega$ that does not contain two symmetrical points, the random variables (r.v.) $\psi(\boldsymbol{\xi})$, $\boldsymbol{\xi} \in S$ are independent.

Finally, we will also use the Gaussian tail distribution defined by

$\forall t \in \mathbb{R}, \quad \Phi(t)=\frac{1}{\sqrt{2 \pi}} \int_{t}^{+\infty} e^{-s^{2} / 2} d s$. 


\subsection{Global Phase Coherence}

As noticed in [30], most of the geometry of an image is encoded in its phase coefficients. In Fig. 1, we reproduce the experiment which consists in exchanging the moduli of two images: as can be seen, the geometry of the image whose phase was kept persists. From there, in an a contrario framework, the authors of [3] define the global phase coherence (GPC) by measuring how much the geometry is affected when the phase information is lost.

More precisely, given $u$ and a random phase function $\psi$ (in the sense of Definition 1), one can define a random real-valued image $u_{\psi}$ by

$\forall \boldsymbol{\xi} \in \Omega, \quad \widehat{u_{\psi}}(\boldsymbol{\xi})=|\hat{u}(\boldsymbol{\xi})| e^{i \psi(\boldsymbol{\xi})}$.

or equivalently, using the reconstruction formula (1), by

$\forall \mathbf{x} \in \Omega, \quad u_{\psi}(\mathbf{x})=\frac{1}{M N} \sum_{\boldsymbol{\xi} \in \Omega}|\hat{u}(\boldsymbol{\xi})| e^{i\langle\mathbf{x}, \boldsymbol{\xi}\rangle+i \psi(\boldsymbol{\xi})}$.

The random image $u_{\psi}$ is the random-phase noise (RPN) associated with $u[36,16]$. Equation (3) can also be written with cosine functions only. For example, if $M$ and $N$ are odd integers (to get rid of Nyquist frequencies), one has

$$
\begin{aligned}
\forall \mathbf{x} \in \Omega, \quad u_{\psi}(\mathbf{x}) & =|\hat{u}(0)|(-1)^{\varepsilon_{0}} \\
+ & \frac{1}{M N} \sum_{\boldsymbol{\xi} \in \Omega_{+}} 2|\hat{u}(\boldsymbol{\xi})| \cos (\psi(\boldsymbol{\xi})+\langle\mathbf{x}, \boldsymbol{\xi}\rangle),
\end{aligned}
$$

where $\varepsilon_{0}=\mathbf{1}_{\psi(0)=\pi}$, and $\Omega_{+}$is a subset of $\Omega \backslash\{0\}$ that contains one point from each pair of symmetrical points of $\Omega$, so that $\Omega=\{0\} \cup \Omega_{+} \cup\left(-\Omega_{+}\right)$is a partition of $\Omega$. This formula shows that the phase randomization shifts the placement of the cosine components of the signal so that some oscillations will appear in the regions where the original image was flat. Thus, it becomes natural to expect the TV to increase greatly after phase randomization. This effect is striking on the one-dimensional example given in Fig. 2. The authors of [3] derive from this observation the following

Definition 2 (Global Phase Coherence [3]) The global phase coherence of an image $u$ is the number

$\operatorname{GPC}(u)=-\log _{10} \mathbb{P}\left(\operatorname{TV}\left(u_{\psi}\right) \leq \mathrm{TV}(u)\right)$.

In other words, the higher the GPC, the smaller the probability for TV to decrease by phase randomization. Notice that this probability can be very small $\left(10^{-1000}\right.$ and even less), and thus out of reach of most computer representations of floating point numbers (arithmetic underflow). This is why the $\log _{10}$ function is introduced in the definition (another reason is the nice interpretation of (minus) the logarithm of a probability in Information Theory). Experimentally, it has been observed that corrupting an image with blur or noise tend to decrease its GPC. Intuitively, when an image $u$ is blurred, its high-frequency components are attenuated, so that the oscillations of the RPN realizations are smoother; therefore, the TV increase entailed by the phase randomization is expected to be less dramatic than in the sharp case. Now, in a noisy image, the flat regions are corrupted (by the noise) with high frequency variations leading to a TV value which is already high, so that the TV increase produced by the phase randomization is smaller than in a clean image. For now, we have no theoretical justification that goes beyond these heuristic remarks, but they will be confirmed by a practical study in Section 4.4 .

The major drawback of Definition 2 is that no closedform formula is available to compute $\operatorname{GPC}(u)$ as an explicit function of $u$, so that one has to use a computationally heavy Monte-Carlo procedure to estimate it. Assuming the distribution of $\operatorname{TV}\left(u_{\psi}\right)$ to be approximately Gaussian, the authors of [3] suggested to approximate $\operatorname{GPC}(u)$ by ("ga" stands for Gaussian approximation)

$\operatorname{GPC}_{g a}(u)=-\log _{10} \Phi\left(\frac{\mu_{0}-\mathrm{TV}(u)}{\sigma_{0}}\right)$

where $\quad \mu_{0}=\mathbb{E}\left(\operatorname{TV}\left(u_{\psi}\right)\right), \quad \sigma_{0}^{2}=\operatorname{Var}\left(\operatorname{TV}\left(u_{\psi}\right)\right)$,

and $\Phi$ is defined by (2). The values of $\mu_{0}$ and $\sigma_{0}$ can be estimated through $N$ Monte-Carlo samples

$\mathrm{TV}\left(u_{\psi}^{(1)}\right), \mathrm{TV}\left(u_{\psi}^{(2)}\right), \ldots, \mathrm{TV}\left(u_{\psi}^{(N)}\right)$

of the r.v. TV $\left(u_{\psi}\right)$, which leads to a numerical approximation of $\operatorname{GPC}(u)$. Unfortunately, due to the fact that each Monte Carlo sample requires the computation of a Fourier transform, the resulting algorithm is quite slow (even with a good $\mathrm{C}$ implementation, it takes about one minute to obtain a merely decent estimate of the GPC of a $512 \times 512$ image on a standard 3Ghz laptop). Let us mention that the Gaussian approximation of $\operatorname{TV}\left(u_{\psi}\right)$ is analyzed theoretically in Appendix A and Appendix B. From a numerical point of view, the quality of the Gaussian approximation can be evaluated by a Monte-Carlo approach. Using $N$ samples of $u_{\psi}$, one can compute $F_{N}$, the empirical estimate of the tail distribution of $\operatorname{TV}\left(u_{\psi}\right)$, and compare it to its Gaussian counterpart $\Phi$. We checked for $N=10,000$ and several different images that $\left\|F_{N}-\Phi\right\|_{\infty}<0.01$. 


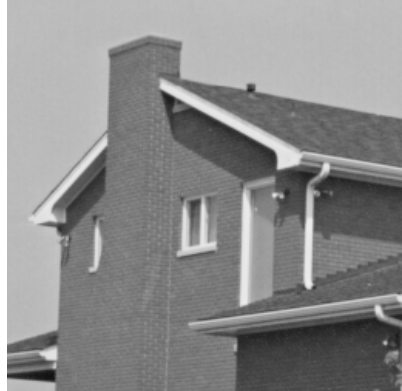

(a) House

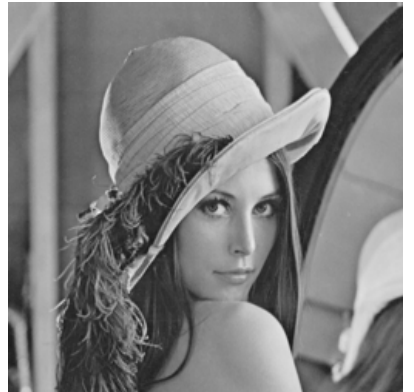

(b) Lena

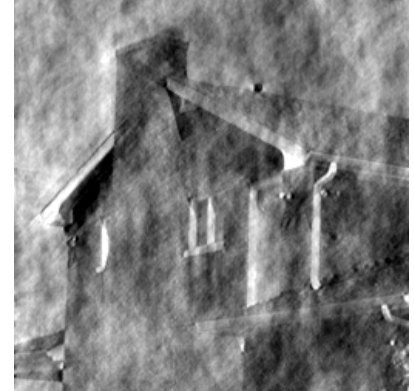

phase of (a) with modulus of (b)

Fig. 1 Phase and perceived geometric content. When an image is built (in Fourier domain) with the phase of an image (a) and the modulus of an image (b), the perceived geometry is that of (a). This famous experiment of Oppenheim and Lim [30] shows that the geometry of an image is mostly encoded in the phase component.

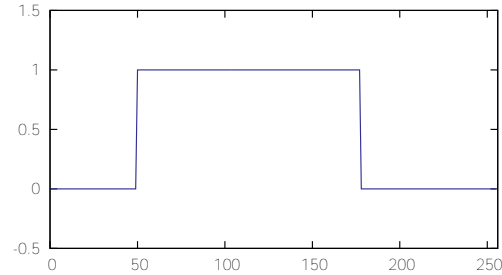

Step function: $\mathrm{TV}=2$

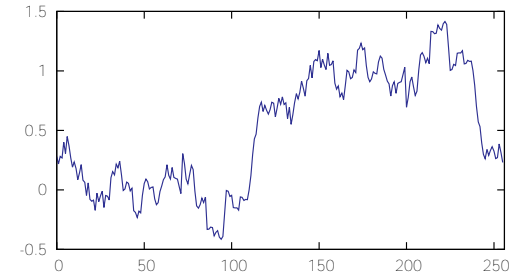

After phase randomization: $\mathrm{TV}=17.4$

Fig. 2 Phase randomization of a step function. Notice the large increase of TV caused by phase randomization.

\subsection{Sharpness Index}

In a later work [4], a new measure of phase coherence was introduced. It was noticed that when replacing the random model $u_{\psi}$ by $u * W$, that is, the convolution of $u$ with a Gaussian white noise $W$, the expectation and variance of $\operatorname{TV}(u * W)$ could be computed explicitly as a function of $u$. Thus, with the same framework as above, one can define

$\mathcal{S I}(u)=-\log _{10} \mathbb{P}(\operatorname{TV}(u * W) \leq \mathrm{TV}(u))$

and, assuming as in [4] that the r.v. $\operatorname{TV}(u * W)$ is approximately Gaussian,

Definition 3 (Sharpness Index [4]) The Sharpness Index (SI) of an image $u$ is

$\mathrm{SI}(u)=-\log _{10} \Phi\left(\frac{\mu-\mathrm{TV}(u)}{\sigma}\right)$

where $\Phi$ is defined by (2),

$\mu=\mathbb{E}(\operatorname{TV}(u * W)), \quad \sigma^{2}=\operatorname{Var}(\operatorname{TV}(u * W))$,

and $W$ is a Gaussian white noise with standard deviation $|\Omega|^{-1 / 2}$ (that is, the r.v. $W(\mathbf{x}), \mathbf{x} \in \Omega$ are independent with distribution $\left.\mathcal{N}\left(0,|\Omega|^{-1}\right)\right)$.
There are several reasons to expect GPC and SI to behave in the same way. First, the corresponding random image models (RPN for GPC, Gaussian for SI) are known to be close, both mathematically (they only differ by a Rayleigh noise on the Fourier modulus) and perceptually (see [16]). Second, it has been noticed experimentally in [4] that the values of $\mu_{0}$ (Equation (6)) and $\mu$ (Equation (9)) were very close in general (a relative error below 1\%). In Appendix A, we confirm this experimental observation by a precise asymptotic result (Theorem 3) based on Berry-Esseen theorem.

The fact that $\mathrm{TV}(u * W)$ is nearly Gaussian (which is used without formal justification in [4]) can again be confirmed by a Monte-Carlo estimation of the distribution of $\operatorname{TV}(u * W)$. We also give an asymptotic proof in Appendix B using a particular central limit theorem devoted to sums of non-independent random variables controlled by a dependency graph.

The great interest of SI over GPC is that it can be computed with explicit formulae instead of a costly Monte-Carlo simulation, as shown in

Theorem 1 ([4]) Let $u: \Omega \rightarrow \mathbb{R}$ be an image and $W: \Omega \rightarrow \mathbb{R}$ be a Gaussian white noise with mean 0 and standard deviation $|\Omega|^{-1 / 2}$. Then the first two moments of $\operatorname{TV}(u * W)$ (see (9)) are given by

$\mu=\left(\alpha_{x}+\alpha_{y}\right) \sqrt{\frac{2}{\pi}} \sqrt{|\Omega|}$, 


$$
\begin{aligned}
\sigma^{2} & =\frac{2}{\pi} \sum_{\mathbf{z} \in \Omega} \alpha_{x}^{2} \cdot \omega\left(\frac{\Gamma_{x x}(\mathbf{z})}{\alpha_{x}^{2}}\right) \\
& +2 \alpha_{x} \alpha_{y} \cdot \omega\left(\frac{\Gamma_{x y}(\mathbf{z})}{\alpha_{x} \alpha_{y}}\right)+\alpha_{y}^{2} \cdot \omega\left(\frac{\Gamma_{y y}(\mathbf{z})}{\alpha_{y}^{2}}\right),
\end{aligned}
$$

where

$$
\begin{aligned}
& \alpha_{x}^{2}=\left\|\partial_{x} \dot{u}\right\|_{2}^{2}=\sum_{(x, y) \in \Omega}|\dot{u}(x+1, y)-\dot{u}(x, y)|^{2}, \\
& \alpha_{y}^{2}=\left\|\partial_{y} \dot{u}\right\|_{2}^{2}=\sum_{(x, y) \in \Omega}|\dot{u}(x, y+1)-\dot{u}(x, y)|^{2}, \\
& \forall t \in[-1,1], \quad \omega(t)=t \arcsin (t)+\sqrt{1-t^{2}}-1,
\end{aligned}
$$

$\Gamma(\mathbf{z})=\left(\begin{array}{ll}\Gamma_{x x}(\mathbf{z}) & \Gamma_{x y}(\mathbf{z}) \\ \Gamma_{y x}(\mathbf{z}) & \Gamma_{y y}(\mathbf{z})\end{array}\right)=\sum_{\mathbf{y} \in \Omega} \nabla \dot{u}(\mathbf{y}) \cdot \nabla \dot{u}(\mathbf{y}+\mathbf{z})^{T}$.

Proof A short proof was given in [4]. In order not to break the discussion about the different definitions of phase coherence, we postpone the complete proof to Appendix C.

Remark: What happens if we replace the TV $\left(l^{1}\right.$-norm of gradient) by the $H^{1}$-norm ( $l^{2}$-norm of gradient) in the definition of SI? With Parseval's formula, one can see that the $H^{1}$-norm only depends on the Fourier modulus, so that it is not affected by the phase randomization. Hence, the corresponding indices obtained with the $H^{1}$-norm are trivial. Considering another $W^{1, p_{-}}$ norm (that is, the $l^{p}$-norm of gradient) could be interesting, but it is likely that the easiest calculations are obtained with $\operatorname{TV}(p=1)$.

\subsection{A Simplified Version of SI}

In [22], we suggested to approximate the denominator of the fraction appearing in (8), which led us to a new index (written $S$ ) that is analytically close to SI but can be computed much faster. We will see empirically later in Section 3 and Section 4 that $S$ also behaves like SI with respect to basic image transformations.

\subsubsection{Definition of $S$}

Lemma 1 The function $\omega$ defined by (12) satisfies

$\forall t \in[-1,1], \quad \frac{1}{2} t^{2} \leq \omega(t) \leq \frac{1}{2} t^{2}+c t^{4}$,

where $c=\frac{\pi-3}{2} \approx 0.0708$ is the optimal (that is, minimal) constant in (13).
Proof One has for all $t \in[-1,1]$,

$\omega^{\prime}(t)=\arcsin (t)=\sum_{n \geq 0} \frac{(2 n) !}{2^{2 n}(n !)^{2}}\left(\frac{1}{2 n+1}\right) t^{2 n+1}$,

(note that the series is absolutely convergent for $|t|=1$ thanks to Stirling's formula). After term-by-term integration, one can write

$\omega(t)=\sum_{n \geq 0} \frac{(2 n) !}{2^{2 n}(n !)^{2}}\left(\frac{1}{2 n+1}\right)\left(\frac{1}{2 n+2}\right) t^{2 n+2}$.

Noticing that $t \mapsto \frac{1}{t^{4}}\left(\omega(t)-\frac{1}{2} t^{2}\right)$ is an even function which is increasing on $[0,1]$, the result follows by taking

$$
\begin{aligned}
c & =\sum_{n \geq 1} \frac{(2 n) !}{2^{2 n}(n !)^{2}}\left(\frac{1}{2 n+1}\right)\left(\frac{1}{2 n+2}\right) \\
& =\lim _{t \rightarrow 1} \frac{\omega(t)-\frac{1}{2} t^{2}}{t^{4}}=\omega(1)-\frac{1}{2}=\frac{\pi-3}{2} .
\end{aligned}
$$

The term (11) can thus be approximated by replacing $\omega(t)$ by $\frac{t^{2}}{2}$. This leads to

$\sigma_{a}^{2}=\frac{1}{\pi}\left(\frac{\left\|\Gamma_{x x}\right\|_{2}^{2}}{\alpha_{x}^{2}}+2 \cdot \frac{\left\|\Gamma_{x y}\right\|_{2}^{2}}{\alpha_{x} \alpha_{y}}+\frac{\left\|\Gamma_{y y}\right\|_{2}^{2}}{\alpha_{y}^{2}}\right)$,

and to

Definition 4 ( $S$ index [22]) The simplified sharpness index associated to an image $u$ is

$S(u)=-\log _{10} \Phi\left(\frac{\mu-\mathrm{TV}(u)}{\sigma_{a}}\right)$,

where $\sigma_{a}$ is given by (14), $\Phi$ by (2), and $\mu$ by (10).

\subsubsection{Fast calculation}

Since the last formula is now free of $\omega$, the index $S$ is, compared to SI, simpler to understand (it only depends on the autocorrelation gradient matrix through its energy) and faster to compute. In Algorithm 1, we can notice that the most costly step is the FFT computation (2.a): once $\hat{u}$ is computed, the FFTs of the two derivatives follow immediately (step 2.b), and the FFTs of the cross-correlation of the derivatives (step 2.c) follow from, e.g.,

$\Gamma_{x x}=\partial_{x} \dot{u} * \widetilde{\partial_{x} \dot{u}} \Rightarrow\left|\widehat{\Gamma_{x x}}\right|=\left|\widehat{\partial_{x} \dot{u}}\right|^{2}$

with the convention that $\widetilde{w}(\mathbf{x})=w(-\mathbf{x})$. In the end, the computation of $S(u)$ requires only 1 FFT, whereas 3 more FFTs are required for $\operatorname{SI}(u)$. In both cases, however, the complexity is the same, $\mathcal{O}(M N \log (M N))$. 


\section{Algorithm 1 : Computation of $S(u)$}

1. Compute the derivatives $\partial_{x} \dot{u}, \partial_{y} \dot{u}$ and deduce their $l^{1}$ and $l^{2}$ norms

$\operatorname{TV}(u), \quad \alpha_{x}=\left\|\partial_{x} \dot{u}\right\|_{2}, \quad \alpha_{y}=\left\|\partial_{y} \dot{u}\right\|_{2}$.

2. Compute (in Fourier domain) the components of the autocorrelation gradient matrix $\Gamma$ :

2.a Compute the FFT $\hat{u}$ of $u$.

2.b Deduce the FFTs of the derivatives using

$$
\begin{gathered}
\left|\widehat{\partial_{x} \dot{u}}(\boldsymbol{\xi})\right|^{2}=2 \sin ^{2}\left(\frac{\pi \xi_{1}}{M}\right)|\hat{u}(\boldsymbol{\xi})|^{2}, \\
\left|\widehat{\partial_{y} \dot{u}}(\boldsymbol{\xi})\right|^{2}=2 \sin ^{2}\left(\frac{\pi \xi_{2}}{N}\right)|\hat{u}(\boldsymbol{\xi})|^{2} .
\end{gathered}
$$

2.c Compute the moduli of the FFTs of $\Gamma_{x x}, \Gamma_{x y}$ and $\Gamma_{y y}$ using

$$
\left|\widehat{\Gamma_{x x}}\right|=\left|\widehat{\partial_{x} \dot{u}}\right|^{2},\left|\widehat{\Gamma_{x y}}\right|=\left|\widehat{\partial_{x} \dot{u}}\right|\left|\widehat{\partial_{y} \dot{u}}\right|,\left|\widehat{\Gamma_{y y}}\right|=\left|\widehat{\partial_{y} \dot{u}}\right|^{2} .
$$

3. Compute $\mu$ and $\sigma_{a}$ with

$$
\begin{aligned}
& \mu=\left(\alpha_{x}+\alpha_{y}\right) \sqrt{\frac{2}{\pi}} \sqrt{M N} \text { and } \\
& \sigma_{a}^{2}=\frac{1}{\pi M N}\left(\frac{\left\|\widehat{\Gamma_{x x}}\right\|_{2}^{2}}{\alpha_{x}^{2}}+2 \cdot \frac{\left\|\widehat{\Gamma_{x y}}\right\|_{2}^{2}}{\alpha_{x} \alpha_{y}}+\frac{\left\|\widehat{\Gamma_{y y}}\right\|_{2}^{2}}{\alpha_{y}^{2}}\right) .
\end{aligned}
$$

4. Finally compute

$S(u)=-\log _{10} \Phi\left(\frac{\mu-\mathrm{TV}(u)}{\sigma_{a}}\right)$

using, if required, the logerf function detailed in [21, Algorithm 1].

\subsubsection{Theoretical comparison with SI}

We here investigate the quality of the approximation of SI by $S$, showing that the fraction

$\mathrm{v}_{a}(u)=\frac{\mu-\operatorname{TV}(u)}{\sigma_{a}}$

is a good approximation of

$\mathrm{v}(u)=\frac{\mu-\mathrm{TV}(u)}{\sigma}$.

\section{Proposition 1 We have}

$0 \leq \frac{\mathrm{v}_{a}(u)-\mathrm{v}(u)}{\mathrm{v}_{a}(u)} \leq 1-\frac{1}{\sqrt{\pi-2}} \approx 0.064$

Proof We first show that

$0 \leq \frac{\sigma^{2}-\sigma_{a}^{2}}{\sigma_{a}^{2}} \leq 2 c=\pi-3 \approx 0.142$
With the expressions of $\sigma$ and $\sigma_{a}$, one can write

$$
\begin{aligned}
\sigma^{2}-\sigma_{a}^{2}=\frac{2}{\pi} \sum_{\mathbf{x} \in \Omega} & \alpha_{x}^{2}\left[\omega\left(\frac{\Gamma_{x x}(\mathbf{x})}{\alpha_{x}^{2}}\right)-\frac{1}{2}\left(\frac{\Gamma_{x x}(\mathbf{x})}{\alpha_{x}^{2}}\right)^{2}\right] \\
+ & 2 \alpha_{x} \alpha_{y}\left[\omega\left(\frac{\Gamma_{x y}(\mathbf{x})}{\alpha_{x} \alpha_{y}}\right)-\frac{1}{2}\left(\frac{\Gamma_{x y}(\mathbf{x})}{\alpha_{x} \alpha_{y}}\right)^{2}\right] \\
+ & \alpha_{y}^{2}\left[\omega\left(\frac{\Gamma_{y y}(\mathbf{x})}{\alpha_{y}^{2}}\right)-\frac{1}{2}\left(\frac{\Gamma_{y y}(\mathbf{x})}{\alpha_{y}^{2}}\right)^{2}\right] .
\end{aligned}
$$

Using Lemma 1, we thus obtain

$\forall t \in[-1,1], \quad 0 \leq \omega(t)-\frac{1}{2} t^{2} \leq c t^{4} \leq c t^{2}$,

which implies

$$
\begin{aligned}
& 0 \leq \sigma^{2}-\sigma_{a}^{2} \\
& \leq \frac{2 c}{\pi} \sum_{\mathbf{x} \in \Omega} \alpha_{x}^{2} \cdot\left(\frac{\Gamma_{x x}(\mathbf{x})}{\alpha_{x}^{2}}\right)^{2}+2 \alpha_{x} \alpha_{y} \cdot\left(\frac{\Gamma_{x y}(\mathbf{x})}{\alpha_{x} \alpha_{y}}\right)^{2} \\
&+\alpha_{y}^{2} \cdot\left(\frac{\Gamma_{y y}(\mathbf{x})}{\alpha_{y}^{2}}\right)^{2}
\end{aligned}
$$

and the right-hand term equals $2 c \sigma_{a}^{2}$, which proves (17). Now, since

$\frac{\mathrm{v}(u)}{\mathrm{v}_{a}(u)}=\left(1+\frac{\sigma^{2}-\sigma_{a}^{2}}{\sigma_{a}^{2}}\right)^{-1 / 2}$,

we get (16) as expected.

Notice that (16) provides a simple universal bound on the relative error $\frac{\mathrm{v}_{a}(u)-\mathrm{v}(u)}{\mathrm{v}_{a}(u)}$. Using the same technique, it could be possible to derive a sharper bound depending on $u$.

To end this Section, let us recall the definitions of $\mathcal{S I}$, SI, and $S$.

$$
\begin{aligned}
\mathcal{S I}(u) & =-\log _{10} \mathbb{P}(\operatorname{TV}(u * W) \leq \operatorname{TV}(u)) \\
\operatorname{SI}(u) & =-\log _{10} \Phi\left(\frac{\mu-\operatorname{TV}(u)}{\sigma}\right) \\
S(u) & =-\log _{10} \Phi\left(\frac{\mu-\operatorname{TV}(u)}{\sigma_{a}}\right)
\end{aligned}
$$

where $\Phi$ is given by (2), $\mu$ by (10), $\sigma$ by (11), and $\sigma_{a}$ by (14).

\section{Mathematical Properties}

\subsection{First properties}

Proposition 2 The functions GPC, SI, SI, $S$ are nonnegative and invariant with respect to affine contrast changes, that is, for $f \in\{\mathrm{GPC}, \mathcal{S I}, \mathrm{SI}, S\}$, one has

$\forall a, b \in \mathbb{R}, a \neq 0, \quad f(a \cdot u+b)=f(u)$. 
Proof These properties directly result from the definitions.

Let us now explore the Fourier representation of the random field $u * W$. Its DFT is $\hat{u} \hat{W}$. Since $W$ is a Gaussian white noise, $\hat{W}$ is a complex Gaussian white noise. In particular, one can write

$\hat{W}(\boldsymbol{\xi})=|\hat{W}(\boldsymbol{\xi})| e^{i \psi(\boldsymbol{\xi})}$

where $\psi$ is a random phase function in the sense of Definition 1. Denoting by $T$ the random image such that $\hat{T}=|\hat{W}|$, one has

$u * W=u_{\varphi+\psi} * T$

where $\varphi+\psi$ is also a random phase. Therefore, in comparison to the phase randomization model, the operation $u \mapsto u * W$ also includes a convolution by an image $T$ whose Fourier transform is $|\hat{W}|$. Following [11], we can say that $T$ is the white noise texton. Proposition 1 of [11] shows that, statistically, $T$ looks like a Dirac mass in zero (up to a factor $\sqrt{\pi} / 2$ ). Hence, one can expect that this convolution will not drastically modify the statistical properties of the model, and, subsequently, that $\operatorname{SI}(u)$ behaves like $\operatorname{GPC}(u)$. Incidentally, the discussion above brings an interesting remark, formulated by the following

Proposition $3 \operatorname{GPC}(u), \mathcal{S I}(u), \operatorname{SI}(u)$, and $S(u)$ only depend on the modulus and the TV of $u$.

Proof For GPC $(u)$ and $\mathcal{S I}(u)$, this is because the distributions of $u_{\psi}$ and $u * W$ only depend on $|\hat{u}|$. For $\operatorname{SI}(u)$ and $S(u)$ this is because the gradient autocorrelation and energy only depend on $|\hat{u}|$.

Thus, all these indices measure the global phase coherence of an image $u$ only by its impact on the TV, in a way (a "scale") that is determined by the modulus of $u$. As we shall see later in Section 4, when an image is filtered by a symmetrical kernel that has a positive Fourier Transform (e.g., a Gaussian kernel), its phase is not changed but the indices above tend to decrease (with the exception of the Dirac image that will be discussed in Section 4.5).

Notice also that since we are using a periodic scheme for TV, these indices take the same values on $u$ and on the periodic translation $\tau_{(a, b)} u$ defined by

$\forall(x, y) \in \mathbb{Z}^{2}, \quad \tau_{(a, b)} u(x, y)=\dot{u}(x-a, y-b)$.

\subsection{Regularity, Analytical Difficulties}

The expression for $\operatorname{SI}(u)$ in Theorem 1 is not defined when $u$ is a constant image. In that case, Equation (7) implies that $\mathcal{S I}(u)$ is zero. It is not a big issue because natural images are never really constant. Apart from these singular points, one can state the following

Proposition 4 Let us introduce

$$
D=\left\{u \in \mathbb{R}^{\Omega},\left\|\partial_{x} \dot{u}\right\|_{2} \neq 0 \text { and }\left\|\partial_{y} \dot{u}\right\|_{2} \neq 0\right\} \text { and }
$$

$D^{\prime}=\left\{u \in \mathbb{R}^{\Omega}, \forall \mathbf{x} \in \Omega, \partial_{x} \dot{u}(\mathbf{x}) \neq 0\right.$ and $\left.\partial_{y} \dot{u}(\mathbf{x}) \neq 0\right\}$.

The functions SI and $S$ are defined and continuous on $D$ and infinitely differentiable on $D^{\prime}$.

Proof Let us consider an image $u \in D$. Thanks to (15) we have $\left\|\Gamma_{x x}\right\|_{2} \neq 0$, and similarly $\left\|\Gamma_{x y}\right\|_{2} \neq 0$ and $\left\|\Gamma_{y y}\right\|_{2} \neq 0$. Consequently, $\sigma$ and $\sigma_{a}$ are non-zero, and $\mathrm{SI}(u)$ and $S(u)$ are well-defined. Moreover, the continuity of SI and $S$ follows from the one of $\alpha_{x}, \alpha_{y}, \Gamma$ and TV. For the second part, we simply notice that the functions $\alpha_{x}, \alpha_{y}, \sigma$ and $\sigma_{a}$ are smooth on $D$, so the singular points of SI and $S$ are those of TV, that is, the images that do not belong to $D^{\prime}$.

The fact that SI have singular points would not be very embarrassing in an optimization perspective. Indeed, several techniques are available to optimize nonsmooth quantities, in particular for convex functions [12]. Unfortunately, the function SI is neither convex nor concave, as shown in Fig. 3. For those reasons, applying classical optimization techniques (like gradient or sub-gradient descent schemes) on SI may not be efficient. We will overcome this difficulty in Section 5 by considering simple generic algorithms relying on stochastic optimization.

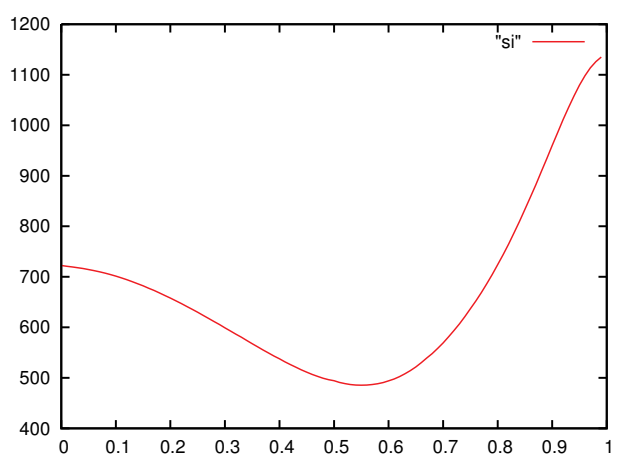

Fig. 3 A one-dimensional profile of SI. This graph of the function $\lambda \mapsto \operatorname{SI}\left(\lambda u_{1}+(1-\lambda) u_{2}\right.$ ) (where $u_{1}$ and $u_{2}$ refer to the images Lena and Barbara respectively) shows that SI is neither convex nor concave. 
3.3 Distribution of GPC on a random phase field

We continue with an explicit statement that generalizes a property mentioned (without proof) in [3].

Proposition 5 If $U$ is a random image such that its phase is uniform (in the sense of Definition 1) and independent of its modulus, then

$\forall t>0, \quad \mathbb{P}(\operatorname{GPC}(U) \geq t) \leq 10^{-t}$.

Furthermore, if conditionally on $|\widehat{U}|$, the r.v. $\operatorname{TV}(U)$ admits a probability density function, then

$\forall t>0, \quad \mathbb{P}(\operatorname{GPC}(U) \geq t)=10^{-t}$,

that is, $10^{-\mathrm{GPC}(U)}$ is uniform on $[0,1]$.

A consequence of (18) is that a texture obtained as the realization of a RPN model or a stationary Gaussian model is expected to have a small GPC value (that is, below 3 or 4 in general), which is in accordance with the fact that such texture models do not carry any phase information. As concerns the hypothesis required for the second part of Proposition 5, it may be satisfied as soon as $U$ is not constant almost surely, but we did not find the proof of such a statement yet.

Proposition 5 can be obtained from the following two Lemmas by considering conditional distributions given $|\widehat{U}|$. Lemma 2 is a general result about cumulative distribution functions that is the key of the proof of Lemma 3.

Lemma 2 If $Y$ is a r.v. and $F(v)=\mathbb{P}(Y \leq v)$, then

$\forall s \in[0,1], \quad \mathbb{P}(F(Y) \leq s) \leq s$,

and the equality holds for all $s$ as soon as $Y$ admits a probability density function.

Proof This is a reformulation of Lemma 1 of [14].

Lemma 3 If $u$ is an image and if $\psi$ is a random phase function (in the sense of Definition 1), then

$\forall t>0, \quad \mathbb{P}\left(\operatorname{GPC}\left(u_{\psi}\right) \geq t\right) \leq 10^{-t}$.

Furthermore, if the r.v. $\mathrm{TV}\left(u_{\psi}\right)$ admits a probability density function, then

$\forall t>0, \quad \mathbb{P}\left(\operatorname{GPC}\left(u_{\psi}\right) \geq t\right)=10^{-t}$.

Proof Let us denote by $F_{u}$ the cumulative distribution function of the r.v. TV $\left(u_{\psi}\right)$, defined by

$\forall t \in \mathbb{R}, \quad F_{u}(t)=\mathbb{P}\left(\mathrm{TV}\left(u_{\psi}\right) \leq t\right)$.

The definition of GPC implies that for any image $u$,

$\operatorname{GPC}(u)=-\log _{10} F_{u}(\mathrm{TV}(u))$.
Since the distribution of $\operatorname{TV}\left(u_{\psi}\right)$ only depends on the modulus of $u$, we have $F_{u}=F_{u_{\chi}}$ for any phase function $\chi$. In particular, if $\psi$ is a random phase function, one can write

$\operatorname{GPC}\left(u_{\psi}\right)=-\log _{10} F_{u_{\psi}}\left(\operatorname{TV}\left(u_{\psi}\right)\right)=-\log _{10} F_{u}\left(\mathrm{TV}\left(u_{\psi}\right)\right)$

so that for all $t>0$,

$\mathbb{P}\left(\operatorname{GPC}\left(u_{\psi}\right) \geq t\right)=\mathbb{P}\left(F_{u}\left(\mathrm{TV}\left(u_{\psi}\right)\right) \leq 10^{-t}\right)$.

Because $F_{u}$ is the cumulative distribution function of $\operatorname{TV}\left(u_{\psi}\right)$, Lemma 2 allows us to conclude that this probability is smaller than $10^{-t}$. The equality case is obtained similarly from the equality case of Lemma 2.

Now we provide a similar result for the approximation $\mathrm{GPC}_{g a}$ of GPC defined in (5).

Proposition 6 Let $u$ be an image and $\psi$ a random phase function (in the sense of Definition 1). Write $\mu_{0}=\mathbb{E}\left(\mathrm{TV}\left(u_{\psi}\right)\right), \sigma_{0}^{2}=\operatorname{Var}\left(\mathrm{TV}\left(u_{\psi}\right)\right)$, and denote by $\widetilde{F}_{u}$ the tail distribution of the normalized r.v.

$T=\frac{\mu_{0}-\mathrm{TV}\left(u_{\psi}\right)}{\sigma_{0}}$,

and by $G_{u}$ the cumulative distribution function of the r.v. $10^{-\mathrm{GPC}_{g a}\left(u_{\psi}\right)}$. If $\mathrm{TV}\left(u_{\psi}\right)$ admits a probability density function then

$\sup _{s \in[0,1]}\left|G_{u}(s)-s\right| \leq \sup _{t \in \mathbb{R}}\left|\widetilde{F}_{u}(t)-\Phi(t)\right|$

Proposition 6 shows that, in terms of the $L^{\infty}$ distance between the cumulative distribution functions, the approximation of $10^{-\mathrm{GPC}_{g a}\left(u_{\psi}\right)}$ by the uniform distribution on $[0,1]$ is at least as good as the Gaussian approximation of $\operatorname{TV}\left(u_{\psi}\right)$.

Proof One can remark that

$$
\begin{aligned}
10^{-\operatorname{GPC}(u)} & =\mathbb{P}\left(\frac{\mu_{0}-\operatorname{TV}\left(u_{\psi}\right)}{\sigma_{0}} \geq \frac{\mu_{0}-\operatorname{TV}(u)}{\sigma_{0}}\right) \\
& =\widetilde{F}_{u}\left(\frac{\mu_{0}-\operatorname{TV}(u)}{\sigma_{0}}\right) .
\end{aligned}
$$

Moreover, we have by definition

$10^{-\mathrm{GPC}_{g a}(u)}=\Phi\left(\frac{\mu_{0}-\mathrm{TV}(u)}{\sigma_{0}}\right)$.

Since $\widetilde{F}_{u}, \mu_{0}$ and $\sigma_{0}$ depend on $u$ only through its modulus, we also have

$$
10^{-\operatorname{GPC}\left(u_{\psi}\right)}=\widetilde{F}_{u}\left(\frac{\mu_{0}-\operatorname{TV}\left(u_{\psi}\right)}{\sigma_{0}}\right)
$$

and $\quad 10^{-\mathrm{GPC}_{g a}\left(u_{\psi}\right)}=\Phi\left(\frac{\mu_{0}-\mathrm{TV}\left(u_{\psi}\right)}{\sigma_{0}}\right)$. 
In particular,

$\left|10^{-\operatorname{GPC}\left(u_{\psi}\right)}-10^{-\operatorname{GPC}_{g a}\left(u_{\psi}\right)}\right| \leq \varepsilon$,

where $\varepsilon=\sup _{t \in \mathbb{R}}\left|\widetilde{F}_{u}(t)-\Phi(t)\right|$. Since we assumed that $\operatorname{TV}\left(u_{\psi}\right)$ admits a probability density function, Lemma 3 ensures that the r.v. $X=10^{-\operatorname{GPC}\left(u_{\psi}\right)}$ follows the uniform distribution on $(0,1)$. So we have almost surely

$\left|X-10^{-\operatorname{GPC}_{g a}\left(u_{\psi}\right)}\right| \leq \varepsilon$,

where $X$ is uniform on $(0,1)$, which implies the inequality (20) for the cumulative distribution functions.

Notice that the result of Proposition 5 does not extend to $\mathcal{S I}$. Actually, one can see empirically in Fig. 4 that it neither extends to SI or $S$. Let us try to understand this by considering the distribution of

$\operatorname{SI}\left(u_{\psi}\right)=-\log _{10} \Phi\left(\frac{\mu-\operatorname{TV}\left(u_{\psi}\right)}{\sigma}\right)$

where $\mu=\mathbb{E}(\operatorname{TV}(u * W))$ and $\sigma=\operatorname{Var}(\operatorname{TV}(u * W))$. Once more, one can assume that $\mathrm{TV}\left(u_{\psi}\right)$ is nearly Gaussian. Concerning the first moment, it has been observed numerically in [4] that $\mathbb{E}\left(\operatorname{TV}\left(u_{\psi}\right)\right) \approx \mathbb{E}(\mathrm{TV}(u * W))$ (this approximation is mathematically investigated in Appendix A). Concerning the variance of TV $\left(u_{\psi}\right)$, however, numerical simulations indicate that it significantly differs (by a factor 7-8 in general [4]) from that of $\mathrm{TV}(u * W)$. A consequence is that the r.v. $G=\frac{\mu-\mathrm{TV}\left(u_{\psi}\right)}{\sigma}$ has a distribution close to $\mathcal{N}\left(0, s^{2}\right)$ for some $s$ that is not close to 1 . Therefore, one cannot expect the distribution of $\Phi(G)=10^{-\mathrm{SI}\left(u_{\psi}\right)}$ to be close to the uniform distribution on $(0,1)$. However, one can see in Fig. 4 that the sharpness values of random phase fields is in general concentrated around 0.3 .

To end this subsection, we mention (without proof) another result concerning the RPN model.

Proposition 7 If $u$ is an image and $\psi$ a discrete random phase field (in the sense of Definition 1), then

$$
\begin{gathered}
\mathbb{P}\left(\operatorname{SI}\left(u_{\psi}\right) \geq \operatorname{SI}(u)\right)=\mathbb{P}\left(\mathcal{S I}\left(u_{\psi}\right) \geq \mathcal{S I}(u)\right) \\
\quad=\mathbb{P}\left(\operatorname{TV}\left(u_{\psi}\right) \leq \operatorname{TV}(u)\right)=10^{-\operatorname{GPC}(u)} .
\end{gathered}
$$

\section{Phase Coherence Indices and No-Reference Quality Assessment}

This Section is devoted to the practical study of the phase coherence indices. Since the computation of $S$ is the fastest of all, we led the experiments on it, but the major part of what follows extends to GPC and SI.

\subsection{Periodization}

The index $S$ deals more with the periodized image $\dot{u}$ than with $u$ itself. Actually, since a periodic translation of $u$ has no effect on $S(u)$, a discontinuity of $u$ on the boundary has the same effect as if it were positioned in the middle of the image. So the index $S$ is affected, and actually biased, by the discontinuities that generally occur between two opposite boundaries of an image. In [3], the authors suggest to compute the phase coherence index not on $u$, but on its periodic component [28]. This operation subtracts from the original image a smooth component that cancels border-to-border discontinuities.

Let us also mention that it is possible to replace in Equation (1) the gradient, the TV, and the autocorrelation by their non-periodic counterparts. It leads to a "Local Sharpness Index" [23] which is a little slower to compute but naturally insensitive to border effects.

\subsection{Quantization}

Another classical operation that can bias the phase coherence is quantization. The gray levels of 8-bits natural images are generally quantized on $\{0,1, \ldots, 255\}$, and this quantization process creates artificially flat regions. The contribution of those regions to the TV is exactly zero, whereas it should be a small (but non-zero) number. To avoid that undesirable effect of quantization, as suggested in [3], before computing these indices, one can apply a sub-pixel translation of vector $(1 / 2,1 / 2)$, with the following definition for the sub-pixel translation of vector $(\alpha, \beta)$,

$\forall \boldsymbol{\xi} \in \Omega, \quad \widehat{\tau_{(\alpha, \beta)} u}(\boldsymbol{\xi})=e^{-2 i \pi\left(\frac{\alpha \xi_{1}}{M}+\frac{\beta \xi_{2}}{N}\right)} \hat{u}(\boldsymbol{\xi})$.

More generally, one could consider the sub-pixel-translation-invariant sharpness index

$\inf _{(\alpha, \beta) \in \mathbb{R}^{2}} S\left(\tau_{(\alpha, \beta)} u\right)$.

Since $\tau_{(a, b)} u$ and $u$ have the same modulus, the vector $(\alpha, \beta)$ corresponding to the minimum value of $S\left(\tau_{(\alpha, \beta)} u\right)$ is actually the one that realizes the maximum value of $\operatorname{TV}\left(\tau_{(\alpha, \beta)} u\right)$. In practice, one can observe that, for most natural images, this vector is usually near $(1 / 2,1 / 2)$, which justifies the use of $\tau=\tau_{(1 / 2,1 / 2)}$ alone.

Another way to avoid the quantization bias on the sharpness indices would be to consider

$\min _{\|v-u\|_{\infty} \leq q / 2} S(v)$

where $q$ is the quantization step ( $q=1$ for integervalued images). Unfortunately, $S$ may have a lot of local 

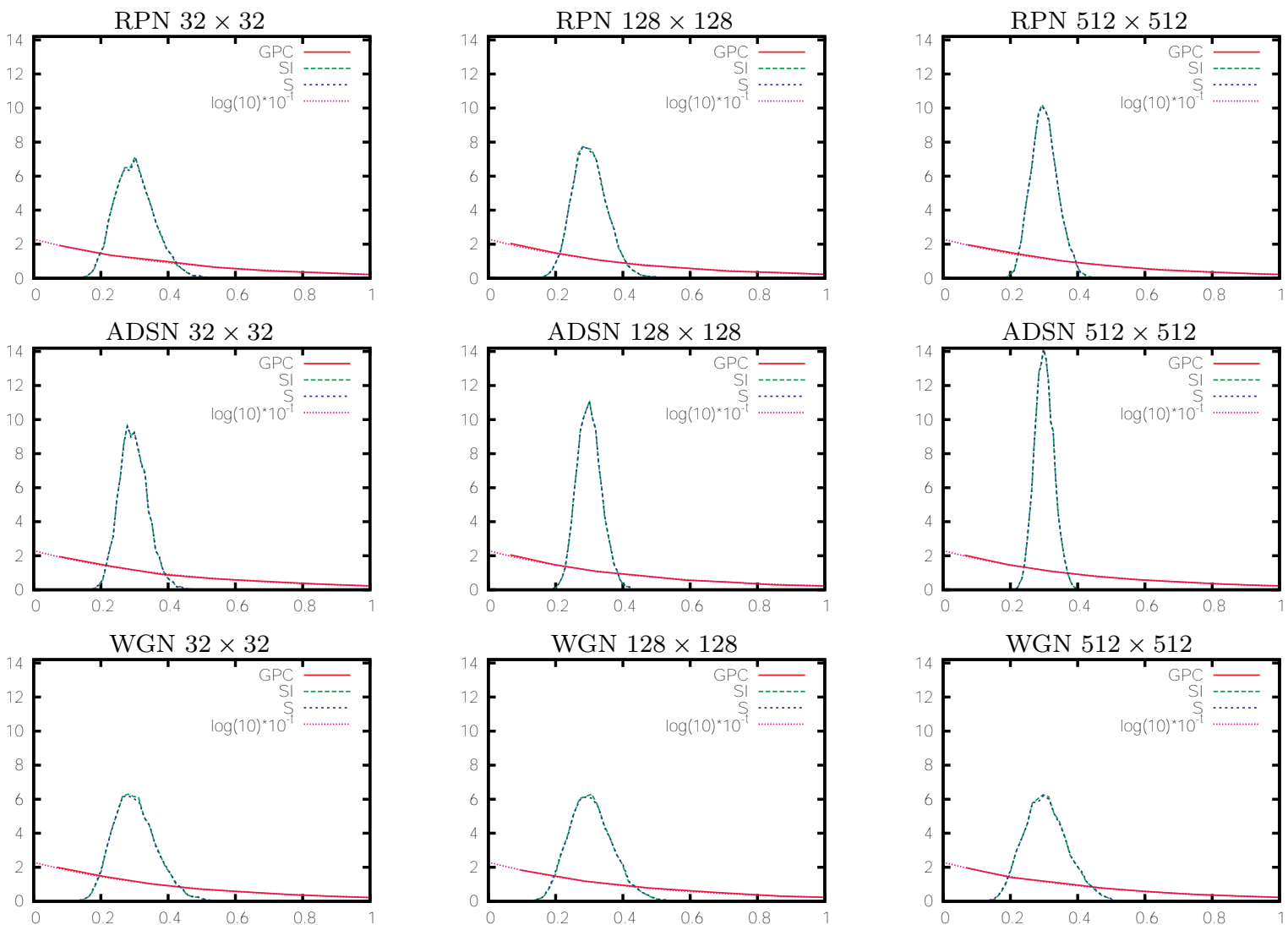

Fig. 4 Phase coherence indices of random phase fields. Each graph represents the estimated distributions (using the same 10,000 samples) of the r.v. $\operatorname{GPC}(U), \operatorname{SI}(U)$ and $S(U)$. The size of the random image $U$ is, respectively, $32 \times 32$ for the left column, $128 \times 128$ for the middle column and $512 \times 512$ for the right column. For the first line, $U$ is the random phase noise (RPN) associated to the image Lena. For the second line, $U$ is the asymptotic discrete spot noise (ADSN) $u * W$ where $u$ is again the image Lena. And for the third line, $U$ is simply a white Gaussian noise (WGN). First, we observe as predicted by Proposition 5 that the distribution of $\operatorname{GPC}(U)$ has density $t \mapsto \log (10) 10^{-t} \mathbf{1}_{t>0}$. Furthermore, we can also observe that the distributions of $\mathrm{SI}(U)$ and $S(U)$ appear similar but that they do not coincide with the one of $\operatorname{GPC}(U)$. Last, we can see that on the RPN and ADSN models, the distributions of SI and $S$ depend on the size of the random field, whereas they apparently do not for the WGN model. However, the mean values of $\operatorname{SI}(U)$ and $S(U)$ remain close to 0.3 .

minima in the neighborhood $\left\{\|v-u\|_{\infty} \leq q / 2\right\}$ of $u$, and it seems difficult to solve (24) by standard optimization techniques.

To end this subsection, we would like to mention that it makes sense to penalize the quantization through the aliasing it produces in the image. The ideal solution to that would be to replace in our construction the simple discrete TV by another TV operator which is invariant by sub-pixel translation. Integrating such an operator (for example, the one suggested in [27]) in our model would be an interesting development. Considering (23) gives an alternative solution which, if $u$ is a natural image, can be approximated by $S(\tau(u))$. Ultimately, the $(1 / 2,1 / 2)$-sub-pixel translation is a precise and efficient solution to avoid the quantization bias.

In the experiments that are presented in the following Sections, before computing the indices SI and $S$, we extracted the periodic component [28] of the image and applied to it a sub-pixel translation of vector $(\mathbf{1} / \mathbf{2}, \mathbf{1} / \mathbf{2})$. Since the DFT of the periodic component of $u$ can be computed with one FFT (see [28]), including these two preprocessing steps yields an overall computation cost of 5 FFTs for SI and 2 FFTs for $S$.

\subsection{Variations of $S$ on natural images}

Before we explore the links between the $S$ index and the perceived sharpness of an image, we give in Fig. 5 some examples of the values obtained for typical natural images. Several observations can be made from these examples, which are confirmed on larger image sets:

- the $S$ index attains higher values for images that present sharp edges and smooth regions at the same time; conversely, out-of-focus images tend to produce relatively low values of $S$; 
- spectrally concentrated textures (in particular, periodic patterns like stripes) lead to surprisingly low values of $S$, even if the texture patterns are made of sharp edges;

- in general, $S$ rapidly increases with the size of images, but since it is very content-dependent, counterexamples (image parts whose $S$-value is greater than the $S$-value of the whole image) can be found.

\subsection{Influence of blur and noise}

In [3] and [4], experiments show that even if the values assigned to an image by GPC and SI can be quite different, both indices decrease when an image is degraded with noise and/or blur. We here check that the same property holds for the $S$ index. Given an initial image $u$, we computed $S\left(\kappa_{\rho} * u+\sigma n\right)$ for several values of $\rho$ (the level of blur) and $\sigma$ (the level of noise), where the Gaussian blur kernel $\kappa_{\rho}$ is defined in Fourier domain by

$\forall \boldsymbol{\xi} \in \Omega, \quad \widehat{\kappa_{\rho}}(\boldsymbol{\xi})=e^{-\frac{1}{2} \rho^{2}\|\boldsymbol{\xi}\|^{2}}$

with $\|\boldsymbol{\xi}\|^{2}=4 \pi^{2}\left(\frac{\xi_{1}^{2}}{M^{2}}+\frac{\xi_{2}^{2}}{N^{2}}\right)$, and $n$ is a realization of a white Gaussian noise with unit variance. The obtained values were then averaged over 10 noise realizations, yielding an estimate of the expectation map

$(\sigma, \rho) \mapsto \mathbb{E}\left(S\left(\kappa_{\rho} * u+\sigma n\right)\right)$

The resulting blur-noise diagrams are displayed in Fig. 6 for the images Barbara and Lighthouse using a representation by level curves (isovalues of $S$ ). We can observe that the $S$ index, like GPC and SI, smoothly decreases with blur and noise, These diagrams are also interesting because they show that $S$ induces an (imagedependent) equivalence between blur and noise. In the case of Barbara for example, we can see that a Gaussian blur of 1.5 pixel is, according to $S$, equivalent to a Gaussian noise with standard deviation 12.6.

\subsection{The Dirac paradox}

Although it seems that for all natural images the value of $S$ decreases when the image is blurred, we found an exceptional case where the opposite phenomenon happens for a very small level of blur. Indeed, if we consider a Dirac image (a single bright pixel on a constant background) and examine the evolution of $S$ when it is blurred by a Gaussian kernel with parameter $\rho$ (as defined in Equation (25)), it happens that $S$ first increases as $\rho$ departs from 0 , then decreases when $\rho$ increases further (Fig. 7). So far, we have not found a theoretical explanation of this phenomenon. We can remark,

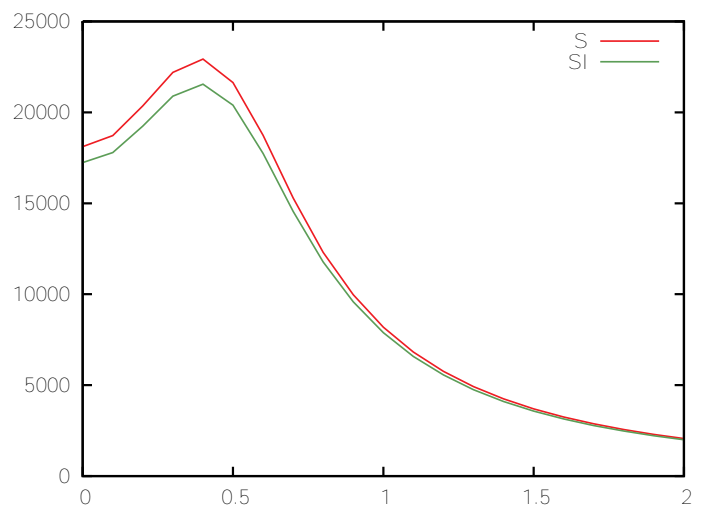

Fig. 7 The Dirac paradox. Evolution of $S$ and SI (vertical axis) for a discrete Dirac image convolved with a Gaussian kernel of width $\rho$ (horizontal axis). Surprisingly, $S$ and SI only decrease after a certain critical value of $\rho$, which shows that the Gaussian kernel that reaches the maximum value of $S$ is not the Dirac, but a slightly blurrier kernel ( $\rho \approx 0.4$ pixels $)$.

however, that it is not really incompatible with the idea that $S$ is linked to image quality and our perception of sharpness: since a Dirac image is aliased, one could consider that a slightly smoother (and hence less aliased) version is sharper (in the sense: more geometrically accurate).

This kind of paradox raises interesting questions linked to the aliasing-ringing-blur trade-off that must face any image reduction (zoom out) algorithm [2]. What is, among the images that represent a single light source (in a sense to be defined), the one that maximizes the value of $S$ ? (the experiment reported in Fig. 7 proves that this is not a Dirac image). What is the unimodal (increasing, then decreasing) one-dimensional signal that maximizes the value of $S$ ? Notice that these questions may be addressed numerically by using the stochastic optimization framework that we describe in Section 5.

\subsection{Sensitivity to ringing, parametric deconvolution}

Suppose that we observe a blurry image $v$ that is the result of the convolution of a clean (unobserved) image $u_{0}$ with a Gaussian kernel (25), plus some (unknown) noise. We can try to invert this blurring process by using the special case of the Wiener filter obtained with $H^{1}$ regularization in a variational setting. Indeed, there is a unique image $u_{\lambda, \rho}$ that minimizes the convex energy

$\left\|\kappa_{\rho} * u-v\right\|_{2}^{2}+\lambda\|u\|_{H^{1}}^{2}$

and it is explicitly given (thanks to Parseval's formula) in Fourier domain by

$\forall \boldsymbol{\xi} \in \Omega, \quad \widehat{u_{\lambda, \rho}}(\boldsymbol{\xi})=\widehat{v}(\boldsymbol{\xi}) \cdot \frac{{\widehat{\kappa_{\rho}}}^{*}(\boldsymbol{\xi})}{\left|\widehat{\kappa_{\rho}}\right|^{2}(\boldsymbol{\xi})+\lambda\|\boldsymbol{\xi}\|^{2}}$ 


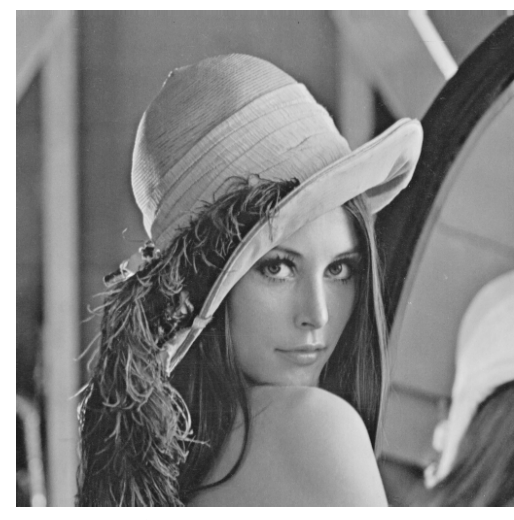

(a) $\mathrm{SI}=943, S=955$

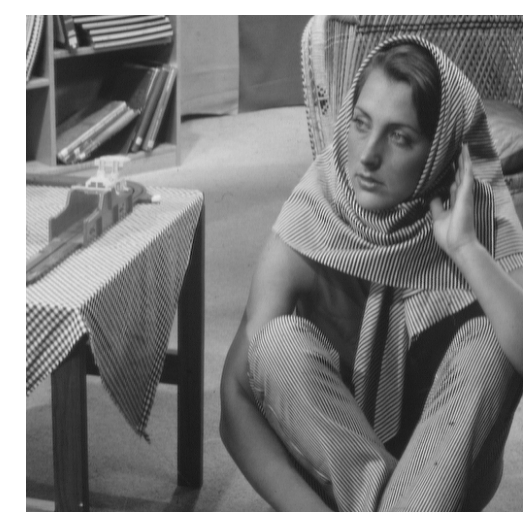

(b) $\mathrm{SI}=679, S=689$

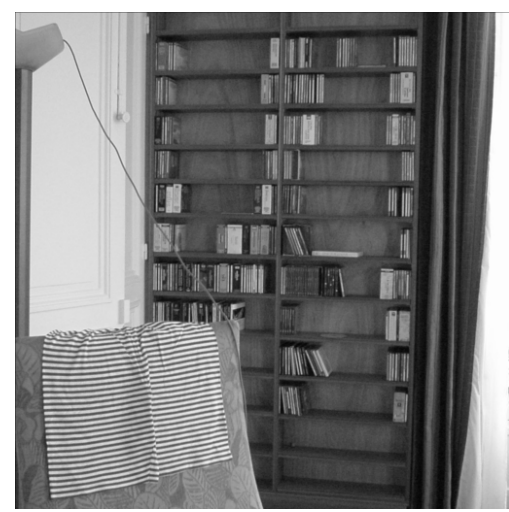

(c) $\mathrm{SI}=115, S=116$

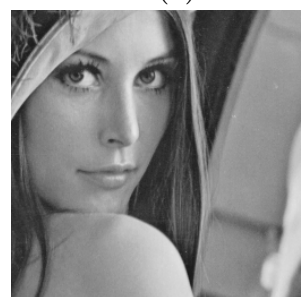

(d) $\mathrm{SI}=155, S=156$

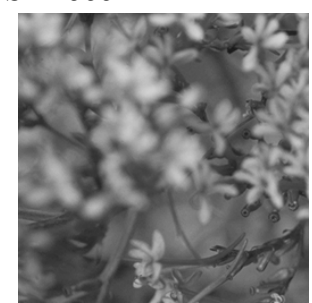

(e) $\mathrm{SI}=31.0, S=31.3$

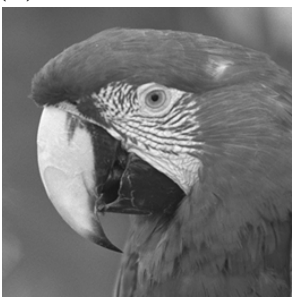

(f) $\mathrm{SI}=709, S=722$

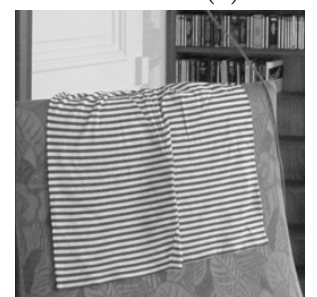

(g) $\mathrm{SI}=4.80, S=4.85$

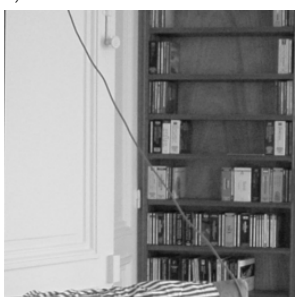

(h) $\mathrm{SI}=333, S=340$

Fig. 5 Examples of values of SI and $S$ for some natural images. One can observe that the values of SI and $S$ are very close, and tend to be small for out-of-focus images like (e) and in the case of a strong high-frequency spectral component (g). Also, the order of magnitude of SI and $S$ grows with the image size (compare the values for the $512 \times 512$ images of the first row to those of the $256 \times 256$ images of the second row), but it may happen that a sub-part of an image has a larger value of $S$ (or SI) than the whole image, as in (c) and (h).
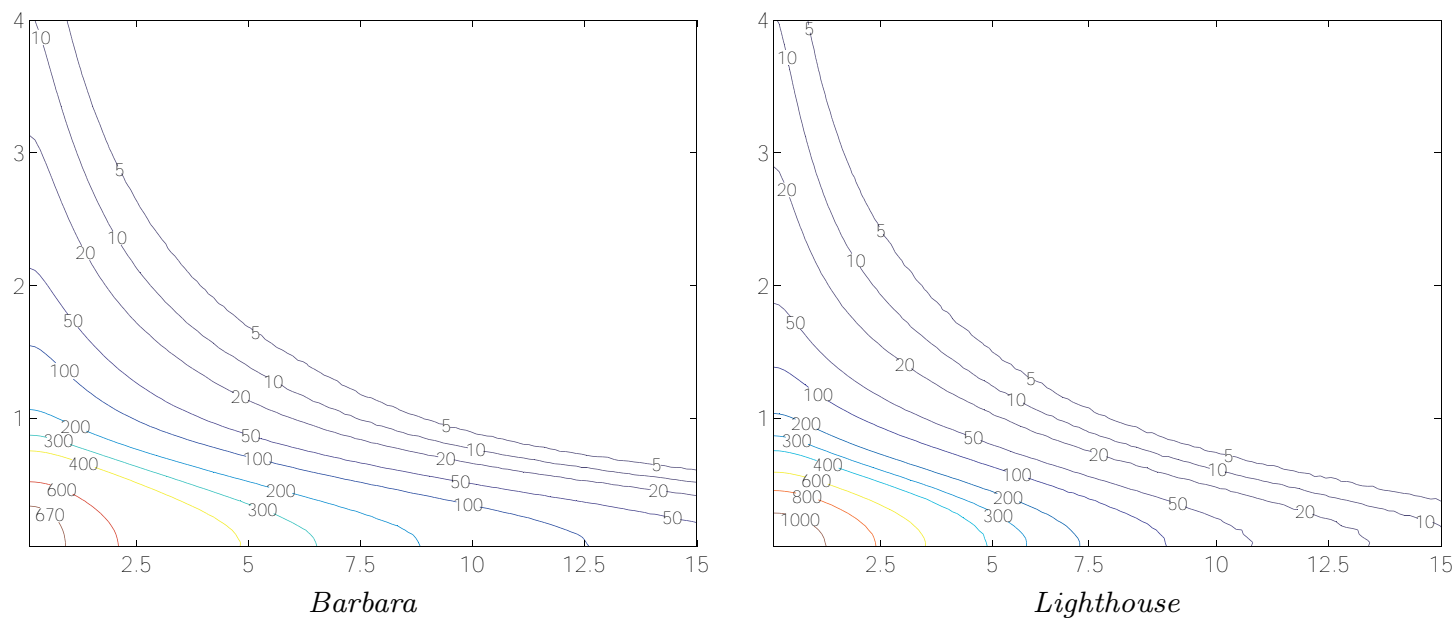

Fig. 6 Blur-noise diagrams. Each diagram displays the isolevel curves of $S$ obtained when a given image (Barbara on the left, Lighthouse on the right) is degraded with a certain amount of blur (vertical coordinate) and noise (horizontal coordinate). As expected, the largest value of $S$ is obtained in each case at the origin (no blur, no noise), and decreases smoothly (in a rather similar way) as the levels of blur and noise increase.

This deconvolution method has two parameters $\lambda$ and $\rho$. The first one $\lambda$, sets the importance of the regularization term $\|u\|_{H^{1}}^{2}$ of (26) in comparison to the fidelity term $\left\|\kappa_{\rho} * u-v\right\|_{2}^{2}$, so that if $\lambda$ increases, the image is more regularized. The balance between fidelity and regularization is an interesting problem which is encountered in several image processing tasks, but we will not discuss it here. We decided to set $\lambda=0.01$ which, in our simulations, always gave satisfying results.

The second parameter $\rho$, however, is critical. If $\rho$ is underestimated, some blur remains; if it is overestimated, spurious oscillations (called ringing) appear. As we can see in Fig. 8, SI and $S$ can be used in a very simple way to design an automatic procedure that se- 

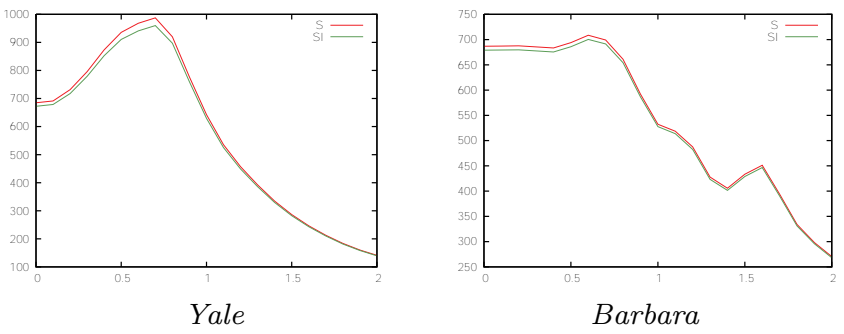

Fig. 8 Blur-ringing trade-offs. These diagrams plot the values of SI (in green) and $S$ (in red) of the $H^{1}$ regularization $u_{\lambda, \rho}$ defined by (27) with $\lambda=0.01$, as functions of the parameter $\rho$ (in pixels) for images Yale (left) and Barbara (right). SI and $S$ attain their maximum value for a very similar value of $\rho$, which corresponds in each case to a good trade-off between blur and ringing for these images (see Fig. 9).

lects an optimal value of $\rho$ (in the sense of the quality of the deconvolved image), because $\operatorname{SI}\left(u_{\lambda, \rho}\right)$ and $S\left(u_{\lambda, \rho}\right)$ are maximal for a value of $\rho$ that corresponds very well to the transition between blur and ringing (see Fig. 9). This is quite a remarkable property, for classical image quality indices (including the metric $Q$ presented below) are not sensitive to ringing artifacts in general (see [5]).

\subsection{Comparison with Zhu and Milanfar's $Q$ metric}

In [40], Zhu and Milanfar proposed a sharpness metric $Q$ based on the singular values of the local gradient field of the image. Given a patch $p$ of the image, they consider the two eigenvalues $s_{1} \geq s_{2} \geq 0$ of the gradient covariance matrix ${ }^{2}$ of $p$, and define from it the coherence $R(p)=\frac{s_{1}-s_{2}}{s_{1}+s_{2}}$ (linked to the anisotropy of the patch $p$ ) and the image content metric $Q(p)=s_{1} R(p)$ (which represents the energy in the local dominant orientation). Then, from a set of nonoverlapping patches, a subset $\mathcal{P}$ of anisotropic patches is extracted by thresholding the coherence $R$, and the metric $Q$ of the whole image is defined as the mean value of $Q(p)$ for $p \in \mathcal{P}$. Notice that when comparing the values of $Q$ on different (possibly noisy, blurred or restored) versions of a particular image, the same set of anisotropic patches must be used. Since $\mathcal{P}$ is extracted from a set of nonoverlapping patches, the computation time for $Q$ is $\mathcal{O}(M N)$.

In particular, Zhu and Milanfar used $Q$ to select an optimal number of iterations in the steering kernel regression (SKR) denoising algorithm of Takeda et al. [35]. We reproduced the same experiment and compared the effects of the $Q$ and the $S$ indices in Fig. 10.

2 The gradient covariance matrix of an image $u$ is the value at $\mathbf{z}=0$ of the gradient autocorrelation matrix $\Gamma$ defined in Theorem 1 .
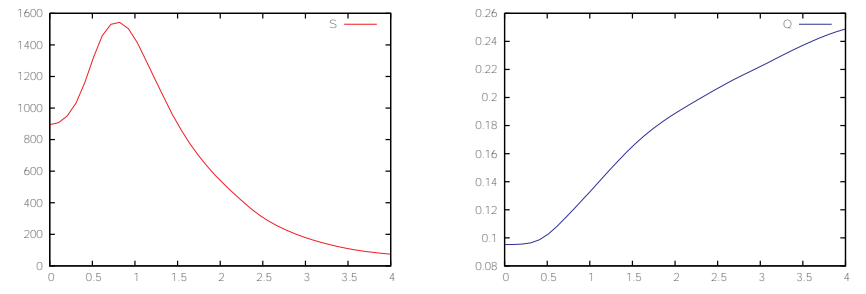

Fig. 11 S versus $\mathbf{Q}$. These diagrams represent the values of $S$ (left) and the values of the metric $Q$ of Zhu and Milanfar (right) computed on the $H^{1}$ regularization $u_{\lambda, \rho}$ defined by (27) with fixed $\lambda=0.01$ and varying $\rho$ (horizontal axis), for Lena image. One can see that $S$ admits an optimal value whereas $Q$ does not. Therefore, contrary to $S$, the metric $Q$ cannot be used for parametric blind deblurring, as it does not consider that ringing artifacts decrease image quality. This limitation of $Q$ is studied more deeply in [25].

Interestingly enough, the global behavior of both indices is the same: as the level of denoising (that is, the number of iterations in [35]) increases, both indices grow, attain a maximal value, then decrease. However, it can be observed that the $S$ index attains its maximum value for a smaller number of iterations (8, versus 14 for $Q)$. This effect is confirmed on other experiments (not displayed here): the $S$ index seems to consider that at some point, the denoising structures left by the SKR algorithm are sharp details and leads to a lower denoising level. This general behavior will be discussed further in Section 4.8: an image process that creates phase-coherent artifacts may increase the $S$ index.

As the sharpness metrics SI and $S$, the $Q$ metric is sensitive to blur and noise. However, it is not sensitive to ringing, so that the parametric deconvolution process described in Section 4.6 cannot be achieved with the $Q$ index, as shown in Fig. 11. This is a crucial difference between these two indices.

\subsection{Perceptual sharpness and Visual Summation}

Even if GPC, SI and $S$ are sensitive to noise, blur and ringing, we should not forget that they were initially designed to measure phase coherence, and that it only appears that they can be interpreted as image quality indices. Thus, contrary to image quality metrics designed on purpose, there is no reason a priori that these indices reflect accurately our visual perception of sharpness. An interesting illustration of this is brought by image compression. For example, JPEG compression is known to produce artificial edges (in particular along the boundaries of the $8 \times 8$ blocks used for DCT), and as these edges require global phase coherence, one can logically expect them to produce high values of GPC, SI and S. Fig. 12 confirms this analysis. Note, how- 

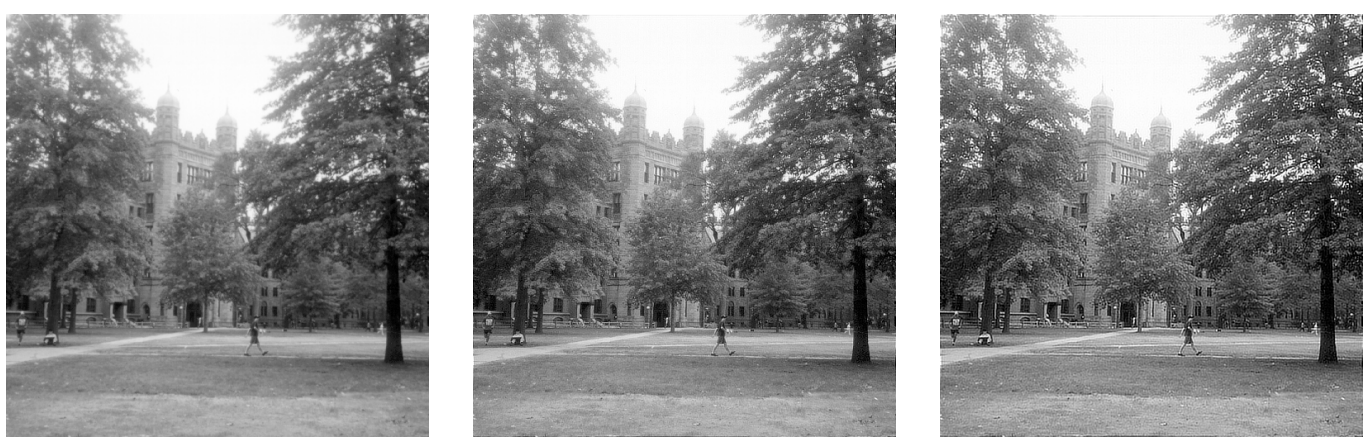

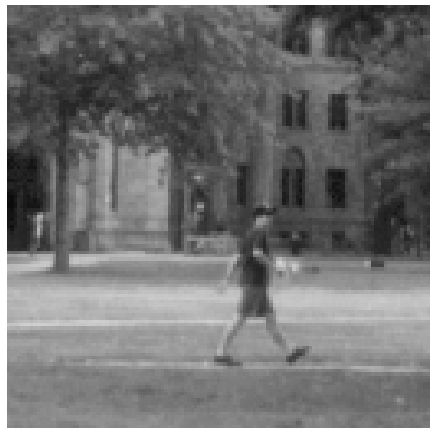

Original

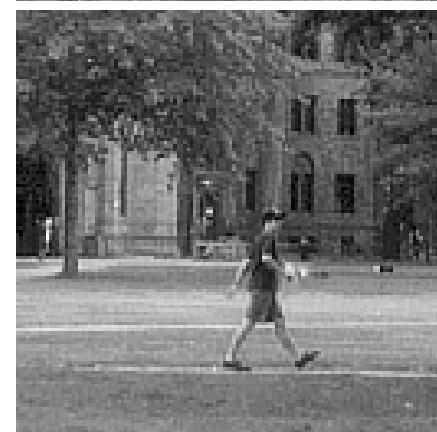

Wiener deconvolution $(\rho=0.7)$

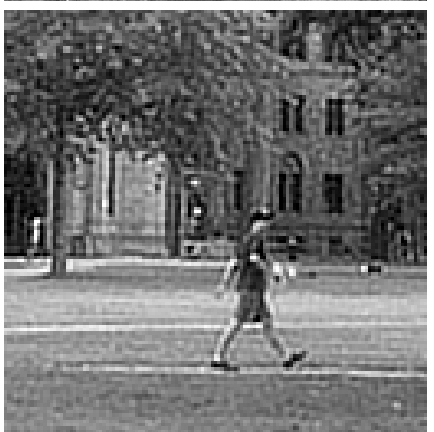

Wiener deconvolution $(\rho=1)$

Fig. 9 Parametric blind deconvolution using sharpness indices. On the first row, we can see the original Yale image (left), and two Wiener- $H^{1}$ deconvolution results obtained with a kernel width of $\rho=0.7$ (middle) and $\rho=1$ (right). Close-up views of these three images are shown on the second row. The value $\rho=0.7$, which maximizes the sharpness indices SI and $S$ (see Fig. 8), corresponds surprisingly well to the desired critical value that rules the transition between blur and ringing.

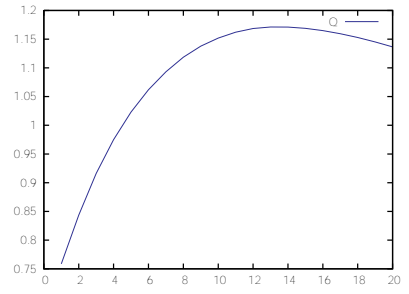

Evolution of $Q$

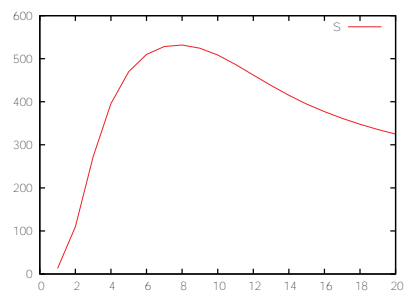

Evolution of $S$

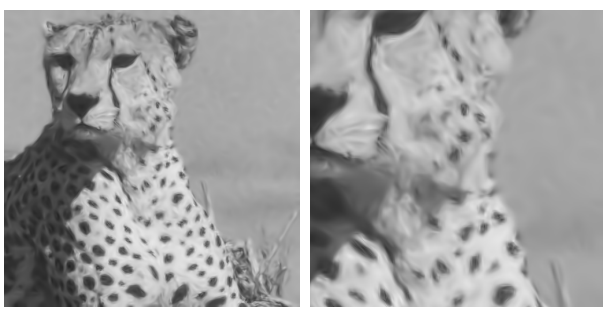

$Q$ optimized, 14 iterations

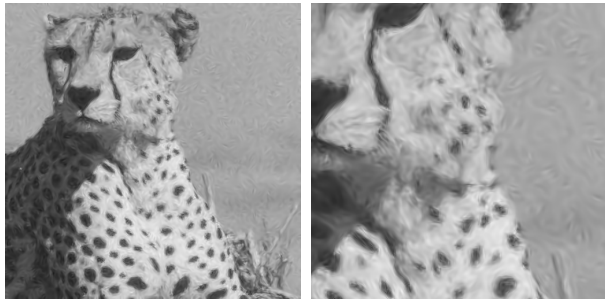

$S$ optimized, 8 iterations

Fig. 10 Parameter selection in SKR denoising: $Q$ versus $S$. The plots on the left report the evolution of $Q$ and $S$ as functions of the number of iterations in the SKR denoising. The input is the image Cheetah corrupted by a white Gaussian noise with standard deviation 18. Both indices are able to select an optimal number of iterations, and the resulting images are shown in the middle column (with some close-up views on the right). Note that the residual phase-coherent artifacts left by the SKR algorithm are considered as sharp by the $S$ index, which thus selects a number of iterations that is significantly smaller. In that particular application, the $Q$ metric is best suited to denoise uniform zones, while the $S$ index leads to better texture preservation. 


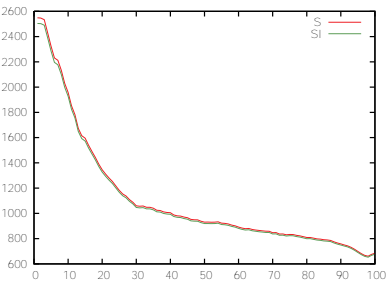

Barbara

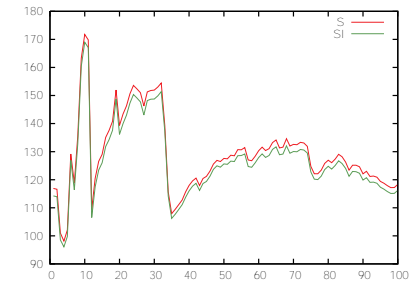

House
Fig. 12 Sharpness indices and JPEG compression. These diagrams show the evolution of SI (in green) and $S$ (in red) when an image (respectively, Barbara on the left, and House on the right) is compressed using the JPEG standard. The horizontal scale refers to the JPEG quality parameter. One can see that $S$ and SI do not reflect our perception of image quality in this case: they increase as the image compression rate increases. This phenomenon, due to the artificial phase coherence brought by the image uncompression scheme, could be avoided by considering instead, for a given compressed image, the minimum sharpness of all possible original images.

ever, that one could probably adapt the sharpness indices we defined to reflect more accurately the quality of compressed images. One possible solution would be to define the sharpness $S_{C}(a)$ of a compressed image $a=C(u)$ (here $C$ denotes the compression operator) by the minimum sharpness found among all possible uncompressed versions of $a$, that is

$S_{C}(a)=\min _{v, C(v)=a} S(v)$.

Such a definition could reflect more accurately our perception of image quality, and would in particular satisfy the desirable property $S_{C}(C(u)) \leq S(u)$ (that is, compression cannot increase image quality).

If we follow the idea of relating the sharpness indices GPC, SI and $S$ to perceptual sharpness, the issue of normalization with respect to image size must be addressed. As we saw in Section 4.3, these indices tend to grow rapidly with the size of an image, which does not really correspond to our visual perception. One possibility to deal with this problem could be to use a "visual summation" principle [37], and define the overall sharpness of an image as the maximal sharpness of all its fixed-size (say, $32 \times 32$ ) sub-parts. A less extreme variant could be to weight the sharpness of each sub-part by some sort of saliency measure. These solutions would solve the size-dependence issue, and thus probably increase the similarity between the proposed indices and our visual perception of sharpness. However, the obtained indices would be analytically more complicated and probably less stable when addressing restoration problems like the blind deblurring application we consider in the next Section.

\section{An Application to Blind Deblurring}

In Section 4.6, we saw that the $S$ index could be used to select a parameter in a deconvolution process. In this Section, we will show that it can drive much more general blind deblurring algorithms. Blind deblurring consists in sharpening an image without knowing precisely the blurring process involved in the image acquisition. We here focus on linear and spatially-invariant blur, which can be modeled by a convolution operator. There is an abundant literature on that subject, and regular advances. We will compare the results we obtain with the method recently proposed by Levin et al. [24], which can produce impressive results.

To design blind deblurring algorithms based on the $S$ index, we will follow the general scheme proposed in [4]. Let us denote by $u_{0}$ the image to recover, by $\varphi$ an unknown convolution kernel and by $n$ an additive noise. Instead of trying to recover the kernel $\varphi$ and then invert the image formation process $u=\varphi * u_{0}+n$, we will select a restoration kernel $k$ that maximizes $S(k * u)$, the sharpness of the restored image $k * u$. In this framework, $k$ can be interpreted as a regularized inverse of $\varphi$ that is supposed to mitigate the effects of the noise. Of course, the linearity of the deblurring process is a limitation of this approach, but as we shall see, a well-chosen linear filter may perform surprisingly well compared to more sophisticated non-linear image transforms. Moreover, linearity has several advantages like stability, computational efficiency, and the fact that deconvolution artifacts (and in particular the effect on noise) are much better understood in the linear case.

\subsection{Remarks on $k \mapsto S(k * u)$}

As mentioned above, the idea underlying the algorithms that will follow is the maximization of the function

$F_{u}: k \mapsto S(k * u)$

on a given set $\mathcal{K}$ of deconvolution kernels. Since the function $S$ is quite singular, it is worth discussing the existence of maxima. First, Proposition 4 ensures that, as soon as the set $\{k * u, k \in \mathcal{K}\}$ does not contain any image which is constant in the $x$ or $y$ direction, $F_{u}$ is continuous on $\mathcal{K}$. Moreover, since $S(\lambda k * u)=S(k * u)$ for any $\lambda \neq 0$, the maximization of $F_{u}$ can be equivalently realized on the bounded set

$\mathcal{K}^{\prime}=\left\{k /\|k\|_{2}, k \in \mathcal{K}\right\}$.

Thus, if $\mathcal{K}^{\prime}$ is closed (which is an easily achievable condition), $F_{u}$ has to be maximized on a compact set and we can thus guarantee the existence of a solution. It 
seems difficult to obtain any guarantee of uniqueness in general (recall that the function $S$ is not concave), but we can at least hope to design algorithms that converge to an interesting local maximum of $F_{u}$. Among them, Algorithm 2 below (a direct adaptation of the algorithm proposed in [4]) is very flexible since it can handle various types of kernels, as we will see in the next Subsections.

\begin{tabular}{|c|}
\multicolumn{1}{c|}{ Algorithm 2 } \\
- Begin with $k=\delta_{0}$ \\
- Repeat $n$ times \\
$\quad \triangleright$ Define $k^{\prime}$ from a random perturbation of $k$ \\
$\quad \triangleright$ If $S\left(k^{\prime} * u\right)>S(k * u)$ then $k \leftarrow k^{\prime}$ \\
- Return $k$ and $k * u$ \\
\hline
\end{tabular}

\subsection{Kernels with compact support}

A first interesting case is the set of symmetric kernels with a fixed support, e.g. a $11 \times 11$ square . One possible perturbation strategy at each iteration consists in adding a random number uniformly distributed in $[-\alpha, \alpha]$ (say, $\alpha=0.05$ ) to a randomly chosen coefficient of the kernel (see [4]). As shown in Fig. 13, this simple stochastic algorithm already gives interesting sharpening results. However, it may also lead to failure cases, in particular when the image contains some high-frequency structured textures [22]. We believe that these failure cases are mostly due to the fact that this set of kernels contains candidates which are not plausible as deconvolution kernels.

\subsection{Kernel with a radial-unimodal Fourier transform}

To cope with the failure cases of fixed support kernels, we suggested in [22] to consider another class of kernels, whose shape is built in Fourier domain by rotating a radial profile defined by $d$ values

$r(0)=1, r(1), r(2), \ldots, r(d-2), r(d-1)=0$.

More precisely, we consider the deconvolution kernel $k_{r}$ defined in Fourier domain by

$\widehat{k_{r}}\left(\xi_{1}, \xi_{2}\right)=L_{r}\left((d-1) \sqrt{2\left(\left(\frac{\xi_{1}}{M}\right)^{2}+\left(\frac{\xi_{2}}{N}\right)^{2}\right)}\right)$,

where $L_{r}:[0, d-1] \rightarrow \mathbb{R}$ denotes the piecewise affine interpolation of $r$. We also suggested to constrain the discrete profile $r$ to be unimodal, which means that there exists a value $m$ such that

$\forall i<m, r(i+1) \geq r(i)$, and $\forall i \geq m, r(i+1) \leq r(i)$
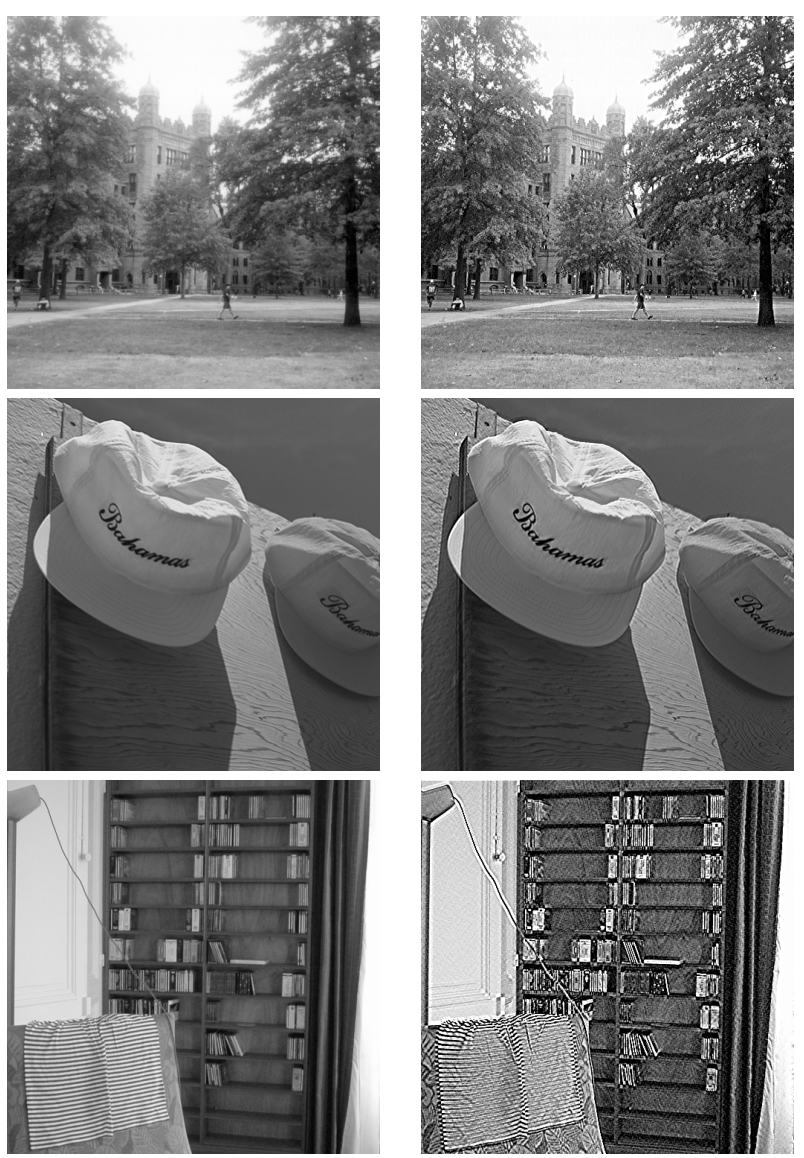

Fig. 13 Blind deblurring results obtained by running Algorithm 2 on the set of $11 \times 11$ kernels. The original (unprocessed) images are shown on the left column (from top to bottom: Yale, Caps (cropped), Room), and the sharpened images are displayed on the right column. In the first two cases, the output image is sharper than the original one and presents a limited quantity of ringing artifacts. However, the result is not satisfactory for the Room image.

The set $U$ of unimodal profiles is rich enough to provide interesting deblurring kernels, and constrained enough to limit distortions in Fourier domain (as large differences in the amplification factor applied to neighboring frequencies tend to produce ringing artifacts). In practice, enforcing the unimodality constraint (by performing a projection on $U$ for example) appeared to be rather inefficient in terms of convergence, and we chose to relax the constraint by incorporating the Euclidean distance $^{3} d(r, U)$ between $r$ and the set $U$ in the objective function. We also decided to constrain the profile $r$ to be smooth with the additional term

$\|r\|_{H^{1}}^{2}=\sum_{i=0}^{d-2}(r(i+1)-r(i))^{2}$.

${ }^{3}$ See Appendix D for the numerical computation of $d(r, U)$. 
Finally, the function to optimize is

$\mathcal{F}_{u}(r)=S\left(k_{r} * u\right)-\lambda_{u m} d(r, U)-\lambda_{\text {reg }}\|r\|_{H^{1}}^{2}$,

where $\lambda_{u m}$ and $\lambda_{\text {reg }}$ are two weighting parameters. The maximization of $\mathcal{F}_{u}$ is realized with Algorithm 3 .

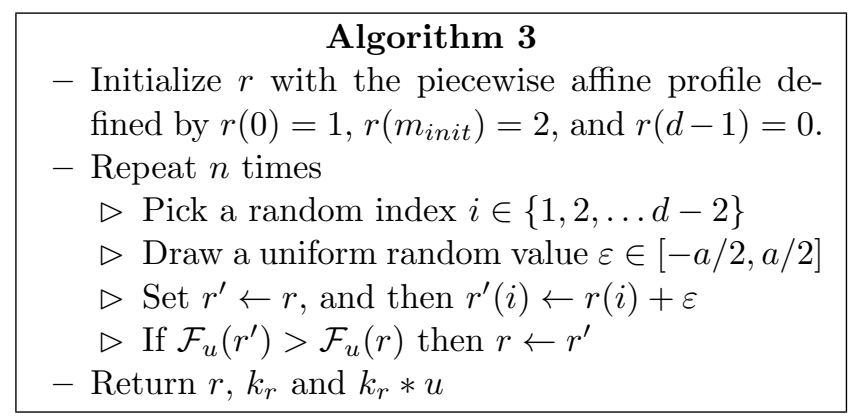

We observed in practice that Algorithm 3 reached a stable state in less than 10000 iterations (which, on a $512 \times 512$ image takes about 4 minutes with a parallel $\mathrm{C}$ implementation using a dual-core processor). Although $\mathcal{F}_{u}$ may have several local maxima, several realizations of the algorithm would always return approximately the same profile $r$, which demonstrates its stability.

Algorithm 3 involves several constants $\left(\lambda_{u m}, \lambda_{\text {reg }}\right.$, $\left.d, m_{\text {init }}, n, a\right)$, but in practice only $\lambda_{r e g}$ is a real parameter. The value $d$ can be set to 20, which achieves a goof trade-off between the dimension of the parameter space and the accuracy of the radial profile. The setup $a=0.1$ led to an efficient proposition strategy in all cases. As mentioned before, the value $n=10000$ seems to be sufficient for convergence, in the sense that the average rate of convergence $\left\|\frac{r_{n e w}-r_{\text {old }}}{r_{\text {old }}}\right\|_{\infty}$ was in general less than $10^{-3}$ after 10000 iterations. To force $r$ to be as close to unimodal as possible, we affected to $\lambda_{u m}$ a high value (10000 in our experiments); we could have made it grow to $+\infty$ in the last iterations. As concerns $m_{\text {init }}$ (the initial mode index), we observed that the different possibilities of initialization (any integer between 1 and $d-2$ ) could lead to two (or three in a few cases) different radial profiles. A systematic strategy would be to try all these indices and select the one leading to the maximum value of $\mathcal{F}_{u}$. In practice, we observed that this maximum value was obtained for an index $m_{\text {init }} \in[d / 4,3 d / 4]$. Besides, in the case where 2 or 3 different radial profiles were obtained (depending on the initialization), we observed that they lead to similar deblurring results. For the sake of simplicity, all the experiments shown in this paper were run with $m_{\text {init }}=d / 4$ (that is, 5$)$.

In Fig. 14, we show some results obtained with Algorithm 3 (for $\lambda_{\text {reg }}=0$ ) on the original images Yale and Barbara (no blur or noise added). In both cases, the resulting image is clearly sharper than the original one and the edges are nicely enhanced, even on the image Barbara which is a difficult case for it contains high-frequency textures.

To assess more precisely the performances of Algorithm 3, we also ran it on artificially degraded images. We transformed each original image $u_{0}$ into a blurry and noisy image

$u=\kappa_{1} * u_{0}+n$,

where $\kappa_{1}$ is the Gaussian kernel (25) obtained for $\rho=1$ and $n$ is a realization of a Gaussian white noise with standard deviation $\sigma=1$. This setup allowed us to build two oracle deblurring filters: the Wiener filter (27) associated to the (supposedly unknown) kernel $\kappa_{1}$, and the oracle radial filter minimizing the expected $l^{2}$ risk, defined by

$k_{0}=\arg \min _{k_{r}} \mathbb{E}\left(\left\|u_{0}-k_{r} *\left(\kappa_{1} * u_{0}+W\right)\right\|^{2}\right)$,

where $W$ is a white Gaussian noise with variance $\sigma^{2}=1$ and the arg min is taken over all kernels $k_{r}$ obtained from an arbitrary radial profile $r$ with $d$ points ${ }^{4}$.

A comparison of the effect of these filters (including Algorithm 3 with several values of the $\lambda_{\text {reg }}$ parameter) is shown on Parrots image in Fig. 15. We can see that Algorithm 3 manages to find a kernel that is close to the Wiener filter associated to the true level of blur $(\rho=1)$. The oracle output reveals slightly more details, but also leaves on the image some undesirable structured noise (which is not costly for the $l^{2}$ risk function that it optimizes). The comparison with [24] is also interesting: compared to Algorithm 3, it manages to clean uniform zones better, but tends to reveal less details in more complex areas (geometric structures or textures). In terms of PSNR (which use is questionable since the original image itself could be noisy and blurry), Algorithm 3 performs better (for $\lambda_{r e g}=10$ ) that [24] and the Wiener oracle, but does not attain the ultimate performance given by the oracle radial filter.

To end this Section, we now discuss the influence of the regularity parameter $\lambda_{\text {reg }}$. As expected, increasing $\lambda_{\text {reg }}$ tends to smooth the radial profile $r$ (see Fig. 15 and 16). One can also see that this regularity prior constrains the overall energy of the kernel, so that when $\lambda_{\text {reg }}$ increases, the kernel values tend to decrease. The Room image (see Fig. 16) is difficult to process because it contains different high-frequency textures that are likely to produce ringing artifacts. In this particular

4 The computation of this oracle kernel is detailed in Appendix E. 


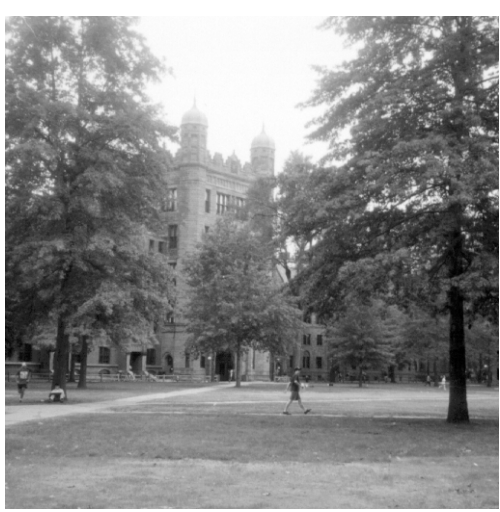

Original

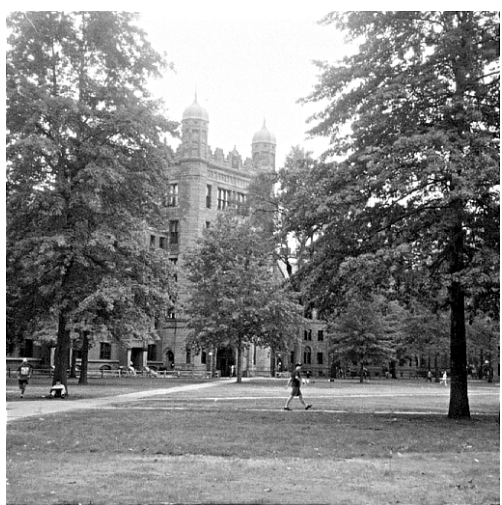

Deblurred

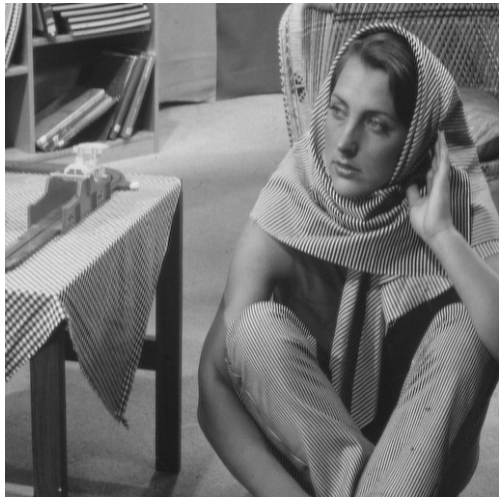

Original

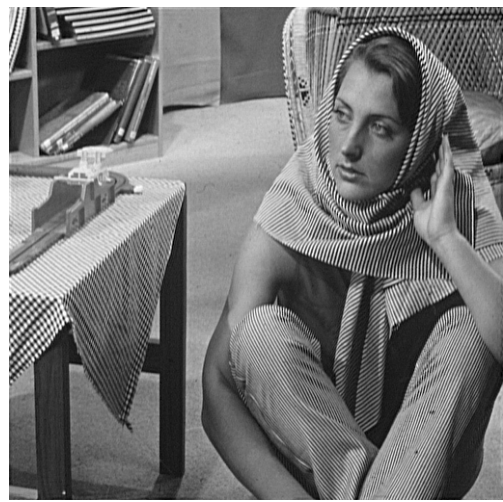

Deblurred

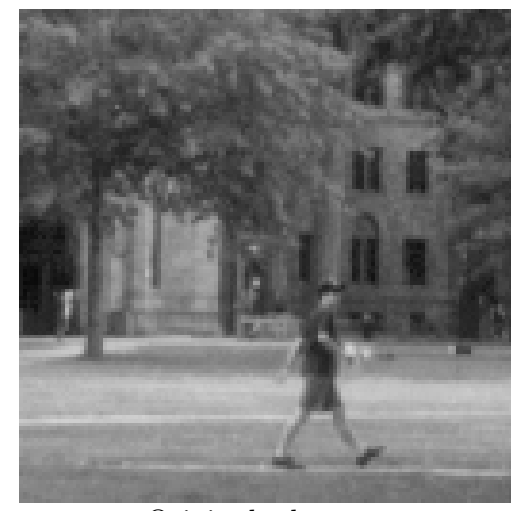

Original, close-up

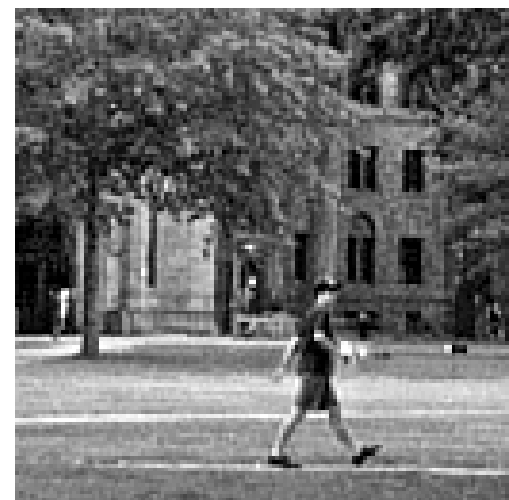

Deblurred, close-up

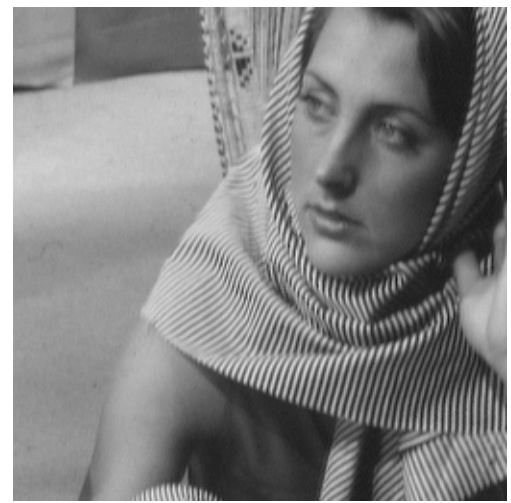

Original, close-up

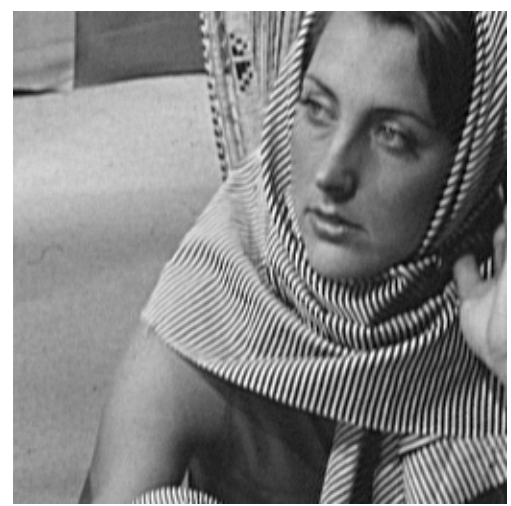

Deblurred, close-up

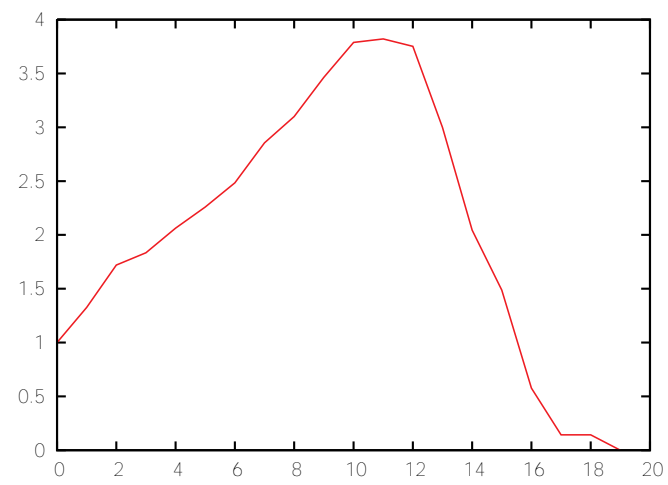

Radial profile $(r)$

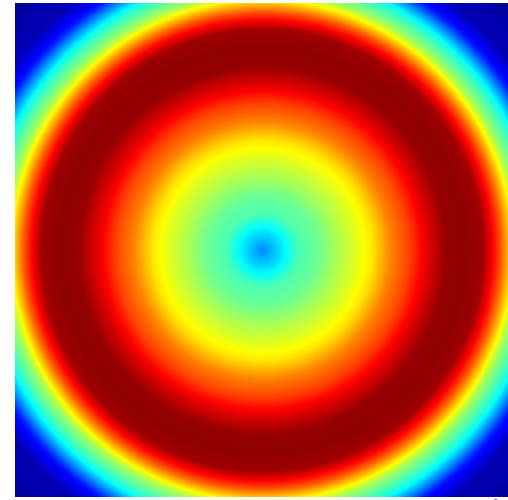

Spectrum of deconvolution kernel $\left(\widehat{k_{r}}\right)$

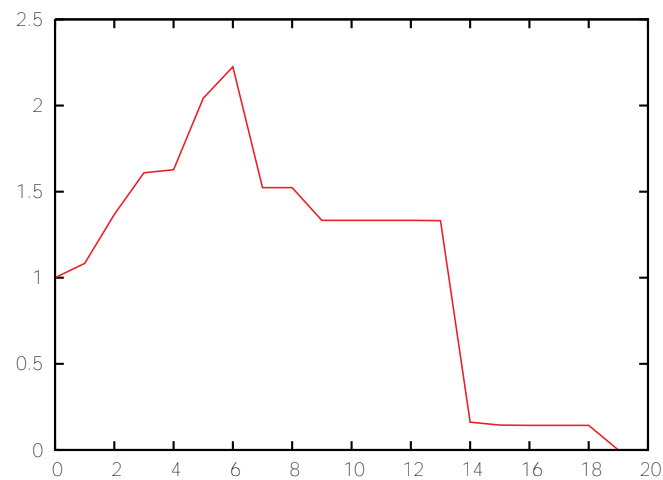

Radial profile $(r)$

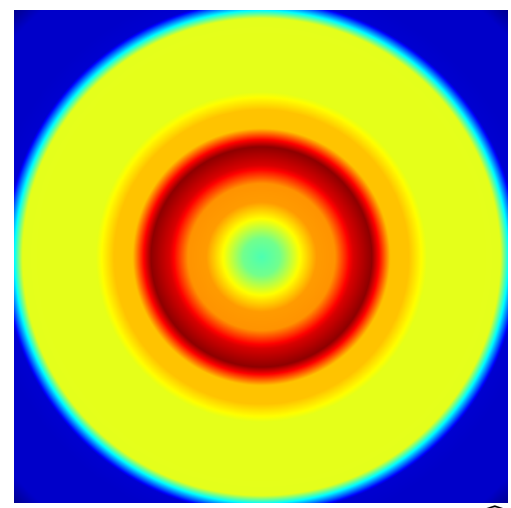

Spectrum of deconvolution kernel $\left(\widehat{k_{r}}\right)$

Fig. 14 Blind deblurring of unprocessed images. Algorithm 3 is applied (with $\lambda_{\text {reg }}=0$, and $n=10000$ iterations) to the images Yale (top 2 rows) and Barbara (bottom rows). In each case, the obtained radial profile $r$ is displayed, as well as the Fourier transform of the corresponding deconvolution kernel $k_{r}$. It is interesting to observe the stability of the proposed algorithm: the deblurred images are much sharper than the original ones, but do not present ringing artifacts or excessive noise amplification. Notice also how the deconvolution kernel adapts itself to each image, leading, in the case of Barbara, to a quite irregular profile. 


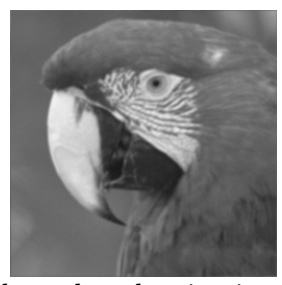

Blurred and noisy input $\mathrm{PSNR}=30.5, \mathrm{~S}=140$

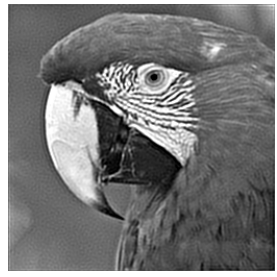

Deblurred $\left(\lambda_{\text {reg }}=0\right)$ $\mathrm{PSNR}=24.5, \mathrm{~S}=440$

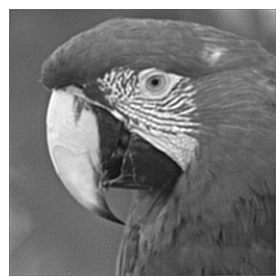

Deblurred $\left(\lambda_{\text {reg }}=10\right)$ $\mathrm{PSNR}=34.2, \mathrm{~S}=394$

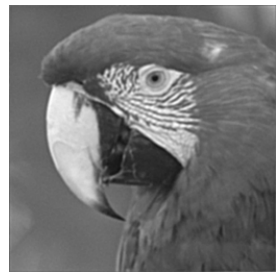

Deblurred $\left(\lambda_{\text {reg }}=100\right)$ $\mathrm{PSNR}=33.8, \mathrm{~S}=300$

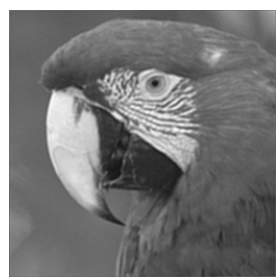

Wiener oracle

$\mathrm{PSNR}=33.7, \mathrm{~S}=316$

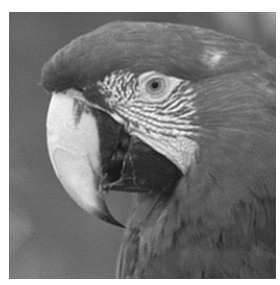

Radial oracle

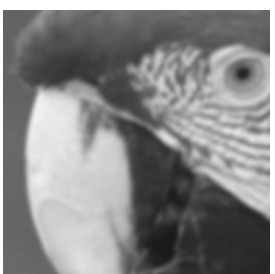

Close-up

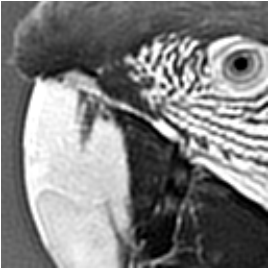

Close-up

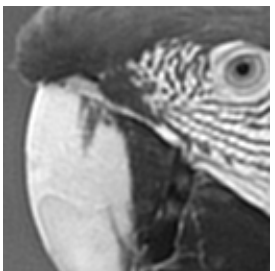

Close-up

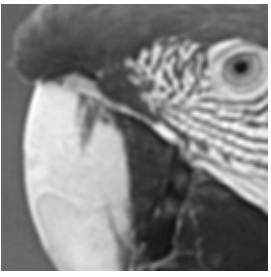

Close-up

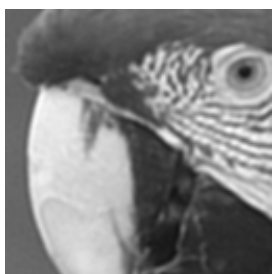

Close-up

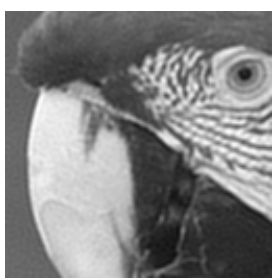

Close-up

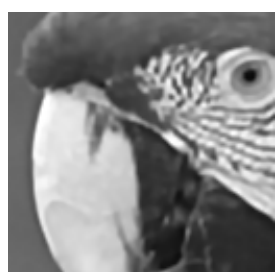

Levin et al. (close-up)

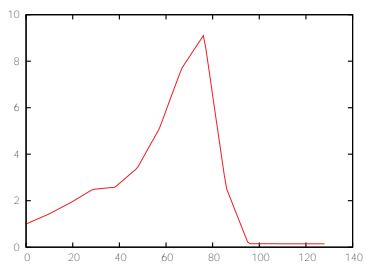

Radial profile

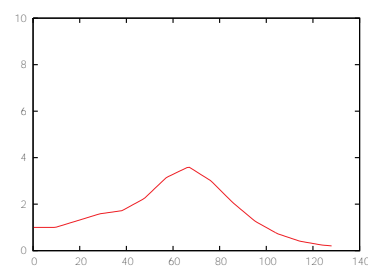

Radial profile

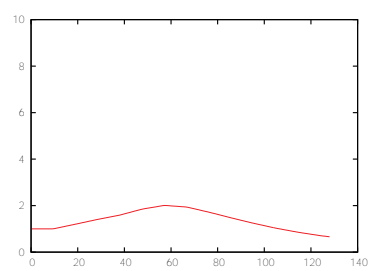

Radial profile

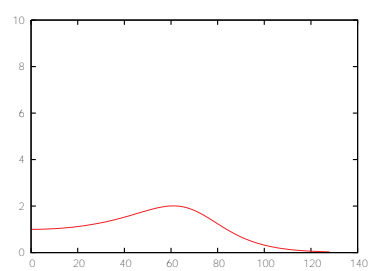

Wiener radial profile

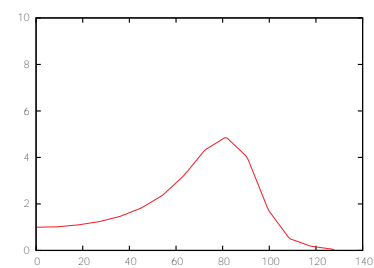

Oracle radial profile

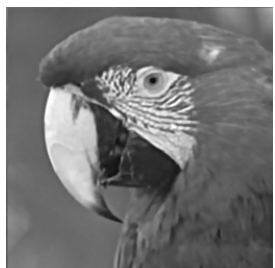

Levin et al.

$\mathrm{PSNR}=27.9, \mathrm{~S}=605$

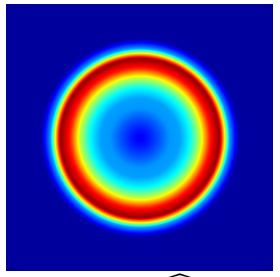

Final $\widehat{k_{r}}$

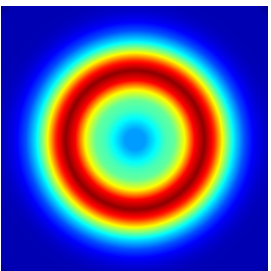

Final $\widehat{k_{r}}$

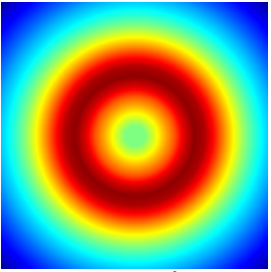

Final $\widehat{k_{r}}$

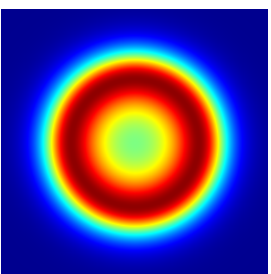

Wiener oracle filter

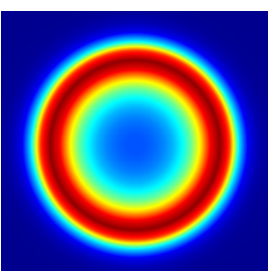

Oracle radial filter

$\mathrm{PSNR}=35.6, \mathrm{~S}=370$

Fig. 15 Blind deblurring of a blurry and noisy version of Parrots. The first row displays the degraded image (used as input), and the deblurred image obtained with Levin et al. algorithm [24]. Each other row is devoted to a different linear algorithm based on a radial kernel (in each case, the radial profile and the Fourier transform of the kernel are displayed). The PSNR values are computed with respect to the original Parrots image. The result obtained with Levin et al. algorithm is cleaner in uniform regions, but slightly less detailed than the one obtained with Algorithm 3 when $\lambda_{r e g}=10$. Notice also the similarity between the filter obtained with $\lambda_{r e g}=10$ and the Wiener oracle filter. Algorithm 3 was used with 10000 iterations. 
case, the regularity constraint is mandatory: the disappointing result obtained for $\lambda_{\text {reg }}=0$ is greatly improved for $\lambda_{r e g}=100$. For the other images we considered (and that are not displayed here), we noticed that the choice $\lambda_{\text {reg }}=100$ always led to visually satisfying results, and $\lambda_{\text {reg }} \in[0,25]$ gave even better results with images that were not too prone to ringing artifacts.

\section{Perspectives}

In this paper, we discussed and compared the phase coherence indices GPC, SI and $S$, and provided some mathematical results as well as several experiments demonstrating their usefulness for no-reference image quality assessment and blind deblurring. The more explicit and simple variants SI and $S$ are clearly an improvement over the original GPC, but many questions remain. The decrease of these indices with respect to noise and blur is easy to check numerically, but a mathematical proof is still to be established. Also, it would be interesting to understand, from an analytical (nonprobabilistic) point of view, why the formulae obtained for SI and $S$ are efficient for image quality assessment and blind deblurring. This could be a way to design non-probabilistic variants, very different from classical analytical regularizers like TV or more generally sparsity-promoting priors. The optimization of $S$ also brings interesting issues, and it seems very likely that the simple iterative stochastic optimization we proposed could be greatly improved, which should increase even further the attractiveness of these indices.

\section{Software resources}

Source codes to compute the GPC, SI and $S$ metrics and images files used in the experiments are freely available on the web page http://www.mi.parisdescartes.fr/ moisan/sharpness/

\section{Appendices}

\section{A Estimation of the mean TV of a RPN}

We saw in Theorem 1 (Equation (10)) that

$\mathbb{E}(\operatorname{TV}(u * W))=\left(\alpha_{x}+\alpha_{y}\right) \sqrt{\frac{2}{\pi}} \sqrt{M N}$.

The right-hand term of (32) appears to be a good approximation of $\mathbb{E}\left(\mathrm{TV}\left(u_{\psi}\right)\right)$, that is, the mean $\mathrm{TV}$ in the RPN model. As noticed in [4], for most images the relative error is around $1 \%$ or below. In this Appendix, we will exhibit an upper bound of the absolute difference.

With the definition of TV, one can write

$\mathbb{E}\left(\operatorname{TV}\left(u_{\psi}\right)\right)=\sum_{\mathbf{x} \in \Omega} \mathbb{E}\left|\partial_{x} \dot{u}_{\psi}(\mathbf{x})\right|+\mathbb{E}\left|\partial_{y} \dot{u}_{\psi}(\mathbf{x})\right|$

so that it is sufficient to show that $\mathbb{E}\left|\partial_{x} \dot{u}_{\psi}(\mathbf{x})\right| \approx \alpha_{x} \sqrt{\frac{2}{\pi M N}}$ for each $\mathbf{x} \in \Omega$. This will follow from a Gaussian approximation of $\partial_{x} \dot{u}_{\psi}(\mathbf{x})$ which implies

$\mathbb{E}\left(\left|\partial_{x} \dot{u}_{\psi}(\mathbf{x})\right|\right) \approx \sqrt{\frac{2}{\pi}} \sqrt{\mathbb{E}\left(\left(\partial_{x} \dot{u}_{\psi}(\mathbf{x})\right)^{2}\right)}$

(notice that the equality holds for a zero-mean Gaussian r.v., as shown by Lemma 4 of Appendix C).

With the Fourier reconstruction formula, one can write that for all $\mathbf{x} \in \Omega$,

$\partial_{x} \dot{u}_{\psi}(\mathbf{x})=\frac{1}{M N} \sum_{\boldsymbol{\xi} \in \Omega}|\hat{u}(\boldsymbol{\xi})| e^{i \psi(\boldsymbol{\xi})} e^{i\langle\mathbf{x}, \boldsymbol{\xi}\rangle}\left(e^{\frac{2 i \pi x_{1}}{M}}-1\right)$.

For any $\mathbf{x} \in \Omega$, the set $\left(e^{i \psi(\boldsymbol{\xi})} e^{i\langle\mathbf{x}, \boldsymbol{\xi}\rangle}\right)_{\boldsymbol{\xi} \in \Omega}$ is a random phase field. It follows that the r.v. $\left|\partial_{x} \dot{u}_{\psi}(\mathbf{x})\right|$ are identically distributed, but they are not independent a priori. This is why we cannot use the central limit theorem directly on the sum $\sum_{\mathbf{x} \in \Omega}\left|\partial_{x} \dot{u}_{\psi}(\mathbf{x})\right|$. Instead we will use a Gaussian approximation of each $\partial_{x} \dot{u}_{\psi}(\mathbf{x})$ in order to derive a bound for the Gaussian approximation of $\sum_{\mathbf{x} \in \Omega}\left|\partial_{x} \dot{u}_{\psi}(\mathbf{x})\right|$.

The Gaussian approximation of $\partial_{x} \dot{u}_{\psi}(\mathbf{x})$ will be precised with a Berry-Esseen theorem. First, to cope with the Hermitian dependence, we have to introduce a subset $\Omega_{+}$of $\Omega$ that contains exactly one point in each pair of symmetrical points, that is, such that

$\Omega \backslash\left\{0, \boldsymbol{\eta}_{x}, \boldsymbol{\eta}_{y}, \boldsymbol{\eta}_{x y}\right\}=\Omega_{+} \cup\left(-\Omega_{+}\right)$

and the union is disjoint. To make the following proof lighter, we will assume that if they exist, the Nyquist coefficients $\hat{u}\left(\boldsymbol{\eta}_{x}\right), \hat{u}\left(\boldsymbol{\eta}_{x y}\right)$, and $\hat{u}\left(\boldsymbol{\eta}_{y}\right)$ are equal to zero (in general, in natural images these coefficients are very small). Then we can write

$u_{\psi}(\mathbf{x})=|\hat{u}(\mathbf{0})|(-1)^{\varepsilon_{0}}+\frac{1}{M N} \sum_{\boldsymbol{\xi} \in \Omega_{+}} 2|\hat{u}(\boldsymbol{\xi})| \cos (\psi(\boldsymbol{\xi})+\langle\mathbf{x}, \boldsymbol{\xi}\rangle)$

and therefore

$u_{\psi}\left(x_{1}+1, x_{2}\right)-u_{\psi}\left(x_{1}, x_{2}\right)=\frac{1}{M N} \sum_{\boldsymbol{\xi} \in \Omega_{+}} X_{\boldsymbol{\xi}}$,

where we set for all $\boldsymbol{\xi} \in \Omega_{+}$,

$$
\begin{aligned}
X_{\boldsymbol{\xi}} & =2|\hat{u}(\boldsymbol{\xi})|\left(\cos \left(\psi(\boldsymbol{\xi})+\langle\mathbf{x}, \boldsymbol{\xi}\rangle+\frac{2 \pi \xi_{1}}{M}\right)-\cos (\psi(\boldsymbol{\xi})+\langle\mathbf{x}, \boldsymbol{\xi}\rangle)\right) \\
& =-4|\hat{u}(\boldsymbol{\xi})| \sin \left(\psi(\boldsymbol{\xi})+\langle\mathbf{x}, \boldsymbol{\xi}\rangle+\frac{\pi \xi_{1}}{M}\right) \sin \left(\frac{\pi \xi_{1}}{M}\right) .
\end{aligned}
$$

Since the $X_{\boldsymbol{\xi}}$ are independent and centered r.v., we can apply the following generalization of Berry-Esseen Theorem (for non identically distributed r.v.):

Theorem 2 (Berry-Esseen, 1942) Let $X_{1}, \ldots, X_{n}$ be independent and centered r.v. in $L^{3}$. Let us denote $\sigma_{i}^{2}=\mathbb{E}\left(X_{i}^{2}\right)$ and $\rho_{i}=\mathbb{E}\left(\left|X_{i}\right|^{3}\right)$. Let $F_{n}$ be the cumulative distribution function of

$\frac{X_{1}+\ldots+X_{n}}{\left(\sigma_{1}^{2}+\ldots+\sigma_{n}^{2}\right)^{1 / 2}}$. 


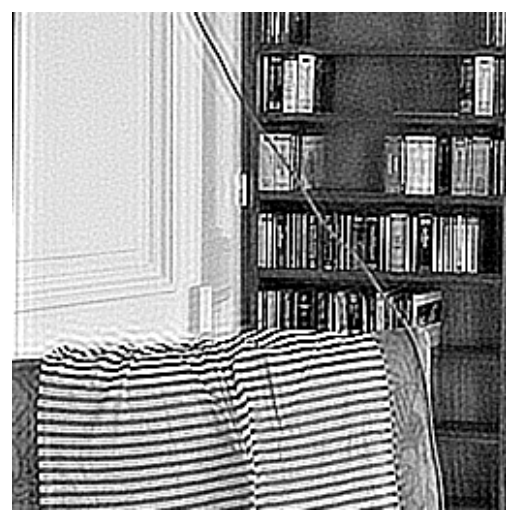

$\lambda_{\text {reg }}=0$

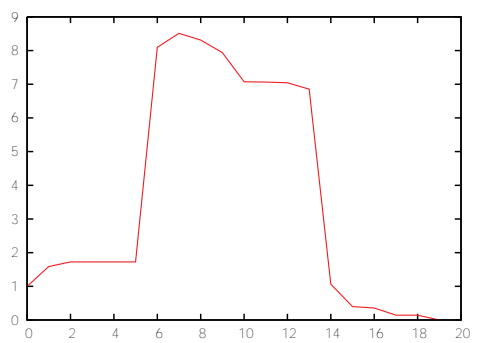

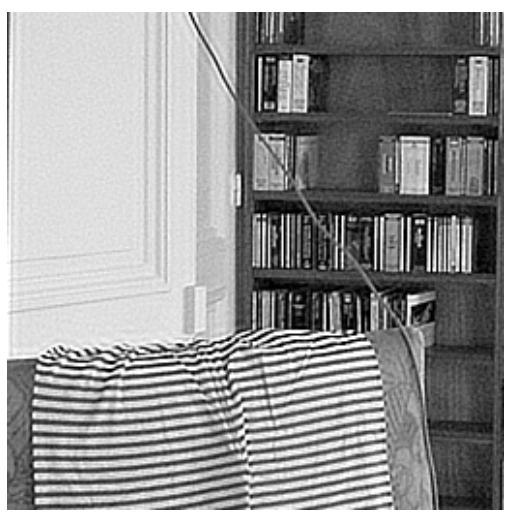

$\lambda_{\text {reg }}=25$

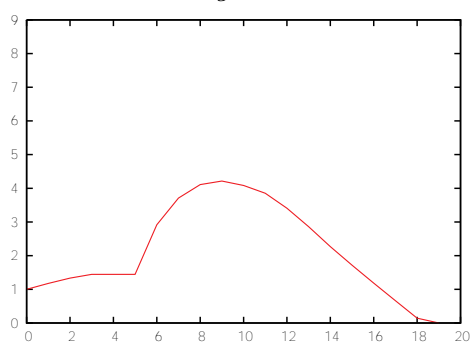

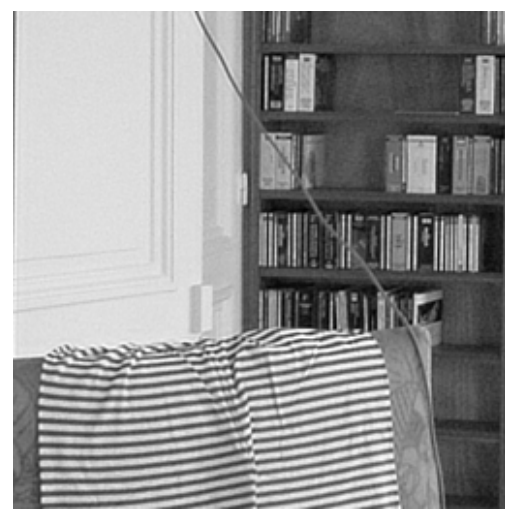

$\lambda_{\text {reg }}=100$

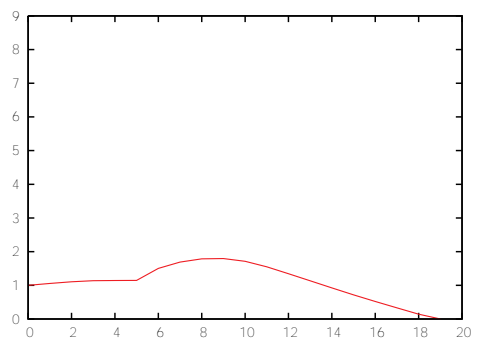

Fig. 16 Blind deblurring of the original Room image for three different levels of regularization of the Fourier profile. On the top row, we display a close-up of the result of the blind deblurring Algorithm 3, which selects (and applies) an optimal radial convolution filter (the corresponding radial profile is shown on the bottom row in each case). The strong ringing artifacts that appear for $\lambda_{r e g}=0$ (left column) are greatly attenuated for $\lambda_{r e g}=25$ (middle) and disappear almost completely for $\lambda_{r e g}=100$. On this kind of images presenting a strong high-frequency content (here, the stripes of the piece of clothing in particular), the parameter $\lambda_{r e g}$ plays a crucial role. Algorithm 3 was used with 10000 iterations.

Then there exists a positive universal constant $C_{0}$ such that $\forall t \in \mathbb{R}, \quad\left|F_{n}(t)-\mathbb{P}(Y \leq t)\right| \leq C_{0} \psi_{0}$

where $Y \sim \mathcal{N}(0,1)$ and $\psi_{0}=\left(\sum_{i=1}^{n} \sigma_{i}^{2}\right)^{-3 / 2}\left(\sum_{i=1}^{n} \rho_{i}\right)$.

Concerning the value of $C_{0}$, some recent papers (e.g. [33]) have shown that the best constant $C_{0}$ is below 0.56 .

Let us apply this theorem to the r.v. $X_{\boldsymbol{\xi}}, \boldsymbol{\xi} \in \Omega_{+}$. Remark that if the r.v. $U$ is uniformly distributed on $[0,2 \pi]$, then $\mathbb{E}\left(\sin ^{2}(U)\right)=\frac{1}{2}$ and $\mathbb{E}\left(|\sin (U)|^{3}\right)=\frac{4}{3 \pi}$. Thus, we have for all $\boldsymbol{\xi} \in \Omega_{+}$,

$\sigma_{\boldsymbol{\xi}}^{2}:=\mathbb{E}\left(X_{\boldsymbol{\xi}}^{2}\right)=8|\hat{u}(\boldsymbol{\xi})|^{2} \sin ^{2}\left(\frac{\pi \xi_{1}}{M}\right)$,

$\rho_{\boldsymbol{\xi}}:=\mathbb{E}\left(\left|X_{\boldsymbol{\xi}}\right|^{3}\right)=\frac{4^{4}}{3 \pi}|\hat{u}(\boldsymbol{\xi})|^{3}\left|\sin \left(\frac{\pi \xi_{1}}{M}\right)\right|^{3}$

Consequently,

$$
\begin{aligned}
\sum_{\boldsymbol{\xi} \in \Omega_{+}} \sigma_{\boldsymbol{\xi}}^{2} & =\sum_{\boldsymbol{\xi} \in \Omega_{+}} 8|\hat{u}(\boldsymbol{\xi})|^{2} \sin ^{2}\left(\frac{\pi \xi_{1}}{M}\right) \\
& =\sum_{\boldsymbol{\xi} \in \Omega} 4|\hat{u}(\boldsymbol{\xi})|^{2} \sin ^{2}\left(\frac{\pi \xi_{1}}{M}\right) \\
& =\sum_{\boldsymbol{\xi} \in \Omega}|\hat{u}(\boldsymbol{\xi})|^{2}\left|e^{\frac{2 i \pi \xi_{1}}{M}}-1\right|^{2}=\left\|\widehat{\partial_{x} \dot{u}}\right\|_{2}^{2}=M N\left\|\partial_{x} \dot{u}\right\|_{2}^{2}
\end{aligned}
$$

and

$\sum_{\boldsymbol{\xi} \in \Omega_{+}} \rho_{\boldsymbol{\xi}}=\frac{4^{4}}{3 \pi} \sum_{\boldsymbol{\xi} \in \Omega_{+}}|\hat{u}(\boldsymbol{\xi})|^{3}\left|\sin \left(\frac{\pi \xi_{1}}{M}\right)\right|^{3}=\frac{128}{3 \pi}\left\|\widehat{\partial_{x} \dot{u}}\right\|_{3}^{3}$.
Hence, noticing that

$\frac{1}{\sqrt{M N}\left\|\partial_{x} \dot{u}\right\|_{2}} \sum_{\boldsymbol{\xi} \in \Omega_{+}} X_{\boldsymbol{\xi}}=\frac{\sqrt{M N}}{\left\|\partial_{x} \dot{u}\right\|_{2}} \partial_{x} \dot{u}_{\psi}(\mathbf{x})$,

and setting

$\psi_{0}=\frac{K(u)}{(M N)^{3 / 2}} \quad$ with $\quad K(u)=\frac{\frac{128}{3 \pi}\left\|\widehat{\partial_{x} \dot{u}}\right\|_{3}^{3}}{\left\|\partial_{x} \dot{u}\right\|_{2}^{3}}$,

Theorem 2 ensures that for all $t \in \mathbb{R}$,

$\left|\mathbb{P}\left(\frac{\sqrt{M N}}{\left\|\partial_{x} \dot{u}\right\|_{2}} \partial_{x} \dot{u}_{\psi}(\mathbf{x}) \geq t\right)-\mathbb{P}(Y \geq t)\right| \leq \frac{C_{0} K(u)}{(M N)^{3 / 2}}$.

Now, we write

$\mathbb{E}\left(\frac{\sqrt{M N}}{\left\|\partial_{x} \dot{u}\right\|_{2}}\left|\partial_{x} \dot{u}_{\psi}(\mathbf{x})\right|\right)=\int_{0}^{+\infty} \mathbb{P}\left(\frac{\sqrt{M N}}{\left\|\partial_{x} \dot{u}\right\|_{2}}\left|\partial_{x} \dot{u}_{\psi}(\mathbf{x})\right| \geq t\right) d t$,

and $\mathbb{E}(Y)=\int_{0}^{+\infty} \mathbb{P}(Y \geq t) d t$,

and we split the integral into two parts : $\int_{0}^{+\infty}=\int_{0}^{A}+\int_{A}^{+\infty}$. Inequality (35) can be integrated between 0 and $A$ to give an upper bound of $\int_{0}^{A}$, whereas the tail $\int_{A}^{+\infty}$ can be treated using Bienaymé-Tchebitchev inequality:

$$
\begin{aligned}
\mathbb{P}\left(\frac{\sqrt{M N}}{\left\|\partial_{x} \dot{u}\right\|_{2}}\left|\partial_{x} \dot{u}_{\psi}(\mathbf{x})\right| \geq t\right) & \leq \frac{1}{t^{2} M N\left\|\partial_{x} \dot{u}\right\|_{2}^{2}} \mathbb{E}\left(\sum_{\boldsymbol{\xi} \in \Omega_{+}} X_{\boldsymbol{\xi}}\right)^{2} \\
& =\frac{1}{t^{2} M N\left\|\partial_{x} \dot{u}\right\|_{2}^{2}} \sum_{\boldsymbol{\xi} \in \Omega_{+}} \sigma_{\boldsymbol{\xi}}^{2}=\frac{1}{t^{2}} .
\end{aligned}
$$


Putting the two terms together, we have for all $A>0$,

$\left|\mathbb{E}\left(\frac{\sqrt{M N}}{\left\|\partial_{x} \dot{u}\right\|_{2}}\left|\partial_{x} \dot{u}_{\psi}(\mathbf{x})\right|\right)-\mathbb{E}(|Y|)\right| \leq \frac{2 C_{0} K(u)}{(M N)^{3 / 2}} A+\frac{2}{A}$,

and then, choosing the best $A$,

$\left|\mathbb{E}\left(\frac{\sqrt{M N}}{\left\|\partial_{x} \dot{u}\right\|_{2}}\left|\partial_{x} \dot{u}_{\psi}(\mathbf{x})\right|\right)-\sqrt{\frac{2}{\pi}}\right| \leq 4 \frac{\sqrt{C_{0} K(u)}}{(M N)^{3 / 4}}$

Therefore, for all $\mathbf{x}$,

$\left|\mathbb{E}\left(\sqrt{M N}\left|\partial_{x} \dot{u}_{\psi}(\mathbf{x})\right|\right)-\alpha_{x} \sqrt{\frac{2}{\pi}}\right| \leq \frac{C_{x}(u)}{(M N)^{3 / 4}}$,

where $C_{x}(u)=4 \sqrt{C_{0}} \sqrt{\frac{\frac{128}{3 \pi}\left\|\widehat{\partial_{x} \dot{u}}\right\|_{3}^{3}}{\left\|\partial_{x} \dot{u}\right\|_{2}}}$.

Recalling that $\alpha_{x}=\left\|\partial_{x} \dot{u}\right\|_{2}$, one has

$$
\left|\mathbb{E}\left(\left\|\partial_{x} \dot{u}_{\psi}\right\|_{1}\right)-\alpha_{x} \sqrt{M N} \sqrt{\frac{2}{\pi}}\right|
$$

$\leq \frac{1}{\sqrt{M N}} \sum_{\mathbf{x} \in \Omega}\left|\mathbb{E}\left(\sqrt{M N}\left|\partial_{x} \dot{u}_{\psi}(\mathbf{x})\right|\right)-\alpha_{x} \sqrt{\frac{2}{\pi}}\right|$

$\leq \frac{1}{\sqrt{M N}} \sum_{\mathbf{x} \in \Omega} \frac{C_{x}(u)}{(M N)^{3 / 4}}$

and thus,

$\left|\mathbb{E}\left(\left\|\partial_{x} \dot{u}_{\psi}\right\|_{1}\right)-\alpha_{x} \sqrt{M N} \sqrt{\frac{2}{\pi}}\right| \leq \frac{C_{x}(u)}{(M N)^{1 / 4}}$.

Finally, we obtain the following

Theorem 3 If $\psi$ is a discrete random phase field, then

$\left|\mathbb{E}\left(\operatorname{TV}\left(u_{\psi}\right)\right)-\left(\alpha_{x}+\alpha_{y}\right) \sqrt{M N} \sqrt{\frac{2}{\pi}}\right| \leq \frac{C_{x}(u)+C_{y}(u)}{(M N)^{1 / 4}}$,

where $\forall a \in\{x, y\}, \quad C_{a}(u)=32 \sqrt{\frac{2 C_{0}}{3 \pi}} \sqrt{\frac{\left\|\widehat{\partial_{a} \dot{u}}\right\|_{3}^{3}}{\left\|\partial_{a} \dot{u}\right\|_{2}}}$.

Theorem 3 provides an explicit bound on the absolute error between the mean TV of a RPN and the exact formula (32) obtained for the associated Gaussian field, but this error bound depends on the considered image and all terms tend to increase with the image size. We can write a normalized inequality by dividing (36) by $\alpha_{x} \sqrt{2 M N / \pi}$, so that

$\left|\frac{\mathbb{E}\left(\left\|\partial_{x} \dot{u}_{\psi}\right\|_{1}\right)}{\alpha_{x} \sqrt{M N}} \sqrt{\frac{\pi}{2}}-1\right| \leq c_{x}(u)$,

where the relative error bound is now

$c_{x}(u):=\frac{32}{(M N)^{3 / 4}} \sqrt{\frac{C_{0}}{3}} \sqrt{\frac{\left\|\widehat{\partial_{x} \dot{u}}\right\|_{3}^{3}}{\left\|\partial_{x} \dot{u}\right\|_{2}^{3}}}=32 \sqrt{\frac{C_{0}}{3}} \sqrt{\frac{\left\|\widehat{\partial_{x} \dot{u}}\right\|_{3}^{3}}{\left\|\widehat{\partial_{x} \dot{u}}\right\|_{2}^{3}}}$

(of course, one would obtain a similar inequality for the $y$ component).

Taking $C_{0}=0.56$, one can compute values of $c_{x}$ for different natural images. For example, $c_{x}(u) \approx 1.025$ for the $512 \times 512$ Lena image, while $c_{x}(u) \approx 0.337$ for the 13 Mpixels
Lotriver image ${ }^{5}$. The bound is quite useless for Lena, and still far from sharp for Lotriver (numerical computations seem to indicate that the true values of the left-hand term of (37) are below $10^{-4}$ for these two images).

Even if it does not provide an accurate error bound, Theorem 3 remains interesting because it indicates that (32) provides the correct asymptotical estimate of the mean TV of a RPN when the image size tends to infinity. Indeed, it has been known for a long time that natural images statistically exhibit a power-law Fourier spectrum (see [8] and other references in [31]), that is,

$|\hat{u}(\boldsymbol{\xi})| \propto|\boldsymbol{\xi}|^{-\alpha}$

in average, where $\alpha$ is a bit larger than 1 in general. Using (38) in the expression of $c_{x}$ above, one easily obtains that for a $R \times R$ image, $c_{x} \propto R^{-1 / 2}$ as $R \rightarrow \infty$, provided that $\alpha<5 / 3$. This suggests that the bound $c_{x}$ tends to decrease to 0 when the size of the considered image increases.

\section{B Gaussian approximation of $\operatorname{TV}(W)$}

We would like to prove that $\operatorname{TV}\left(u_{\psi}\right)$ and $\operatorname{TV}(u * W)$ approximately (or asymptotically) follow Gaussian distributions. Unfortunately, as we already said in the previous Appendix, we cannot apply a classical central limit theorem because the r.v. appearing in the TV formula are not independent. These dependencies introduce a lot of difficulties and this is why we shall here focus on a much simpler problem, that is, the asymptotical distribution of $\mathrm{TV}(W)$ (which is the TV of the Gaussian model in the particular case $u=\delta_{0}$ ).

Proposition 8 Let $\left(\Omega_{n}\right)_{n>0}$ be a sequence of rectangular domains of $\mathbb{Z}^{2}$ such that $\left|\bar{\Omega}_{n}\right| \rightarrow \infty$ when $n$ tends to $\infty$, and let $\left(W_{n}(\mathbf{x})\right)_{\mathbf{x} \in \Omega_{n}}$ be a set of i.i.d. r.v. with distribution $\mathcal{N}\left(0,\left|\Omega_{n}\right|^{-1 / 2}\right)$. Then one has

$\mathrm{TV}\left(W_{n}\right)-\mathbb{E}\left(\mathrm{TV}\left(W_{n}\right)\right) \stackrel{d}{\longrightarrow} \mathcal{N}\left(0, \sigma^{2}\right)$, where

$\mathbb{E}\left(\operatorname{TV}\left(W_{n}\right)\right)=\frac{4\left|\Omega_{n}\right|^{1 / 2}}{\sqrt{\pi}}$ and $\sigma^{2}=\frac{8}{\pi}\left(\omega(1)+6 \cdot \omega\left(\frac{1}{2}\right)\right)$.

To prove this result, we will use the central limit theorem given in [18], which applies to a set of r.v. whose dependencies are controlled through their dependency graph.

Definition 5 ([18]) A graph $\Gamma$ is a dependency graph for a set of r.v. if the following two conditions are satisfied:

1. There exists a one-to-one correspondence between the r.v. and the vertices of the graph.

2. If $V_{1}$ and $V_{2}$ are two disjoint sets of vertices of $\Gamma$ such that no edge of $\Gamma$ has one endpoint in $V_{1}$ and the other in $V_{2}$, then the corresponding sets of r.v. are independent.

Now we can recall the

Theorem 4 (Janson [18]) Suppose, for each integer $n$, that $\left(X_{n, i}\right)_{i=1, \ldots, N_{n}}$ is a set of r.v. satisfying $\left|X_{n, i}\right| \leq A_{n}$ a.s. for all $i$. Suppose further that $\Gamma_{n}$ is a dependency graph for this set and let $M_{n}$ be the maximal degree 6 of $\Gamma_{n}$ (unless $\Gamma_{n}$ has no edges at all, in which case we set $\left.M_{n}=1\right)$.

5 This image is available on the web site http://www.mi. parisdescartes.fr/ moisan/sharpness/

6 We recall that the maximal degree of a graph is the maximal number of edges incident to a single vertex. 
Let $S_{n}=\sum_{i=1}^{N_{n}} X_{n, i}$ and $\sigma_{n}^{2}=\operatorname{Var}\left(S_{n}\right)$. If there exists an integer $m$ such that

$\left(\frac{N_{n}}{M_{n}}\right)^{1 / m} \frac{M_{n} A_{n}}{\sigma_{n}} \rightarrow 0 \quad$ as $\quad n \rightarrow \infty$

then $\frac{S_{n}-\mathbb{E}\left(S_{n}\right)}{\sigma_{n}} \rightarrow \mathcal{N}(0,1)$ in distribution as $n \rightarrow \infty$.

First, we will clarify the remark following this theorem in [18]. It states that we can replace the boundedness hypothesis

$\forall n, \quad \forall i, \quad\left|X_{n, i}\right| \leq A_{n} \quad$ a.s.

by $\frac{M_{n}}{\sigma_{n}^{2}} \sum_{i=1}^{N_{n}} \mathbb{E}\left(X_{n, i}^{2} \mathbf{1}_{\left|X_{n, i}\right|>A_{n}}\right) \rightarrow 0 \quad$ as $n \rightarrow \infty$.

Indeed, assume that (40) is true. We use the truncation argument suggested in [18] and set

$X_{n, i}^{T}=X_{n, i} \mathbf{1}_{\left|X_{n, i}\right| \leq A_{n}}$,

$S_{n}^{T}=\sum_{i=1}^{N_{n}} X_{n, i}^{T}, \quad$ and $\quad\left(\sigma_{n}^{T}\right)^{2}=\operatorname{Var}\left(S_{n}^{T}\right)$.

It is clear that the variables $X_{n, i}^{T}$ have the same dependency degree than the $X_{n, i}$. We will see that (39) is still true for $\sigma_{n}^{T}$ so that Janson's Theorem will give

$\frac{S_{n}^{T}-\mathbb{E}\left(S_{n}^{T}\right)}{\sigma_{n}^{T}} \stackrel{d}{\longrightarrow} \mathcal{N}(0,1)$.

But first let us explain how we control the residual sum. One can write

$$
\begin{aligned}
& \frac{S_{n}-\mathbb{E}\left(S_{n}\right)}{\sigma_{n}}-\frac{S_{n}^{T}-\mathbb{E}\left(S_{n}^{T}\right)}{\sigma_{n}} \\
= & \frac{1}{\sigma_{n}} \sum_{i=1}^{N_{n}}\left(X_{n, i} \mathbf{1}_{\left|X_{n, i}\right|>A_{n}}-\mathbb{E}\left(X_{n, i} \mathbf{1}_{\left|X_{n, i}\right|>A_{n}}\right)\right) .
\end{aligned}
$$

For a fixed $n$, setting

$T_{i}=X_{n, i} \mathbf{1}_{\left|X_{n, i}\right|>A_{n}}-\mathbb{E}\left(X_{n, i} \mathbf{1}_{\left|X_{n, i}\right|>A_{n}}\right)$

(which again have a dependency degree smaller than $M_{n}$ ) and writing $i \sim j$ if $T_{i}$ and $T_{j}$ are not independent, one can write

$$
\begin{aligned}
\mathbb{E}\left(\left(\sum_{i} T_{i}\right)^{2}\right) & =\sum_{i, j} \mathbb{E}\left(T_{i} T_{j}\right) \\
& =\sum_{i} \sum_{j \sim i} \mathbb{E}\left(T_{i} T_{j}\right) \\
& \leq \frac{1}{2} \sum_{i} \sum_{j \sim i} \mathbb{E}\left(T_{i}^{2}\right)+\mathbb{E}\left(T_{j}^{2}\right) \\
& =\frac{1}{2} \sum_{i} \sum_{j \sim i} \mathbb{E}\left(T_{i}^{2}\right)+\frac{1}{2} \sum_{j} \sum_{i \sim j} \mathbb{E}\left(T_{j}^{2}\right) \\
& \leq\left(M_{n}+1\right) \sum_{i} \mathbb{E}\left(T_{i}^{2}\right) \\
& \leq 2 M_{n} \sum_{i} \mathbb{E}\left(T_{i}^{2}\right),
\end{aligned}
$$

which gives

$$
\begin{aligned}
& \mathbb{E}\left(\frac{1}{\sigma_{n}^{2}}\left(\sum_{i=1}^{N_{n}} X_{n, i} \mathbf{1}_{\left|X_{n, i}\right|>A_{n}}-\mathbb{E}\left(X_{n, i} \mathbf{1}_{\left|X_{n, i}\right|>A_{n}}\right)\right)^{2}\right) \\
\leq & 2 \frac{M_{n}}{\sigma_{n}^{2}} \sum_{i=1}^{N_{n}} \operatorname{Var}\left(X_{n, i} \mathbf{1}_{\left|X_{n, i}\right|>A_{n}}\right) \\
\leq & 2 \frac{M_{n}}{\sigma_{n}^{2}} \sum_{i=1}^{N_{n}} \mathbb{E}\left(X_{n, i}^{2} \mathbf{1}_{\left|X_{n, i}\right|>A_{n}}\right) .
\end{aligned}
$$

Therefore, (40) gives that

$$
\frac{S_{n}-\mathbb{E}\left(S_{n}\right)}{\sigma_{n}}-\frac{S_{n}^{T}-\mathbb{E}\left(S_{n}^{T}\right)}{\sigma_{n}} \stackrel{L^{2}}{\longrightarrow} 0 .
$$

To conclude, it remains to show that $\frac{\sigma_{n}^{T}}{\sigma_{n}} \rightarrow 1$ as $n$ tends to $\infty$. Indeed, it is thus equivalent to check condition (39) for $\sigma_{n}$ or $\sigma_{n}^{T}$ so that we are able to apply Janson's theorem to obtain

$$
\frac{S_{n}^{T}-\mathbb{E}\left(S_{n}^{T}\right)}{\sigma_{n}^{T}} \stackrel{d}{\longrightarrow} \mathcal{N}(0,1) .
$$

Moreover it implies that the distributional convergence of $\frac{S_{n}^{T}-\mathbb{E}\left(S_{n}^{T}\right)}{\sigma_{n}^{T}}$ is equivalent to the one of $\frac{S_{n}^{T}-\mathbb{E}\left(S_{n}^{T}\right)}{\sigma_{n}}$. To show that $\sigma_{n}$ and $\sigma_{n}^{T}$ are equivalent, notice that (41) and the reverse Minkowski inequality (in $L^{2}$ ) give

$\left\|\frac{S_{n}-\mathbb{E}\left(S_{n}\right)}{\sigma_{n}}\right\|_{L^{2}}-\left\|\frac{S_{n}^{T}-\mathbb{E}\left(S_{n}^{T}\right)}{\sigma_{n}}\right\|_{L^{2}} \rightarrow 0$,

which is exactly

$1-\frac{\sigma_{n}^{T}}{\sigma_{n}} \rightarrow 0$.

Finally, putting together (41), (42), (43), we obtain that

$$
\frac{S_{n}-\mathbb{E}\left(S_{n}\right)}{\sigma_{n}} \stackrel{d}{\longrightarrow} 0 \text {. }
$$

Let us now get into the details of the application to the TV of a white Gaussian noise. For $\mathbf{x} \in \Omega_{n}$, we will set

$Z_{n, \mathbf{x}}=\left|\dot{W}_{n}(x+1, y)-\dot{W}_{n}(x, y)\right|+\left|\dot{W}_{n}(x, y+1)-\dot{W}_{n}(x, y)\right|$,

so that $\operatorname{TV}\left(W_{n}\right)=\sum_{\mathbf{x} \in \Omega_{n}} Z_{n, \mathbf{x}}$. With these notations, we will be able to apply Janson's theorem on this sum with $M_{n}=6$. Indeed, for a fixed $\mathbf{x}=(x, y) \in \Omega_{n}$, the variables $\dot{W}_{n}(x+1, y), \dot{W}_{n}(x, y+1)$ and $\dot{W}_{n}(x, y)$ appear in $Z_{n, \mathbf{x}}$. These two variables also appear in $Z_{n,(x-1, y)}, Z_{n,(x-1, y+1)}$, $Z_{n,(x, y-1)}, Z_{n,(x+1, y-1)}, Z_{n,(x+1, y)}, Z_{n,(x, y+1)}$, and do not appear in any other $Z_{n, \mathbf{x}}, \mathbf{x} \in \Omega_{n}$. That is why we can set $M_{n}=6$.

Next, to apply the theorem, we also need to know the variance of the sum. It is actually independent of $n$ and given by Theorem 1:

$\sigma^{2}=\sigma_{n}^{2}=\operatorname{Var}\left(\operatorname{TV}\left(W_{n}\right)\right)=\frac{8}{\pi}(\omega(1)+6 \cdot \omega(1 / 2))$.

Notice that the theorem also gives

$\mathbb{E}\left(\operatorname{TV}\left(W_{n}\right)\right)=\frac{4}{\sqrt{\pi}}\left|\Omega_{n}\right|^{1 / 2}$. 
Now, it remains to find a sequence $A_{n}$ which satisfies both (39) and (40). Since in our case $M_{n}$ and $\sigma_{n}$ are constant, we must find $A_{n}$ and an $m$ such that

$\left|\Omega_{n}\right|^{1 / m} A_{n} \rightarrow 0$ and $\sum_{\mathbf{x} \in \Omega_{n}} \mathbb{E}\left(Z_{n, \mathbf{x}}^{2} \mathbf{1}_{\left|Z_{n, \mathbf{x}}\right|>A_{n}}\right) \rightarrow 0$

as $n \rightarrow \infty$. Since all the $Z_{n, \mathbf{x}}$ follow the Gaussian distribution with standard deviation $2\left|\Omega_{n}\right|^{-1 / 2}$, the second condition is equivalent to

$\mathbb{E}\left(Z^{2} \mathbf{1}_{|Z|>A_{n}\left|\Omega_{n}\right|}\right) \rightarrow 0$.

Hence, it suffices to find $A_{n}$ and an $m$ such that

$\left|\Omega_{n}\right|^{1 / m} A_{n} \rightarrow 0$ and $A_{n}\left|\Omega_{n}\right| \rightarrow \infty$.

We can take $m=3$ and $A_{n}=\left|\Omega_{n}\right|^{-1 / 2}$. The two conditions are satisfied, and with Janson's theorem we obtain the result of Proposition 8.

Remark: One can point out that we applied a powerful central limit theorem in order to prove a very specific case. In fact, one can adapt the preceding proof to show that, as soon as $u$ has compact support in $\Omega_{n}$ with $\left|\Omega_{n}\right| \rightarrow \infty$, we have normal convergence of $\mathbb{E}(\operatorname{TV}(u * W))$ after centralization and normalization.

\section{Proof of Theorem 2.1}

Before proving Theorem 1, let us give two lemmas about Gaussian random vectors.

Lemma 4 Let $X$ be a Gaussian r.v. with zero mean and variance $\sigma^{2}$. Then $\mathbb{E}(|X|)=\sigma \sqrt{\frac{2}{\pi}}$.

Proof Since $X \sim \mathcal{N}\left(0, \sigma^{2}\right)$, one can write

$$
\begin{aligned}
E(|X|) & =\frac{1}{\sigma \sqrt{2 \pi}} \int_{-\infty}^{+\infty}|x| e^{-\frac{x^{2}}{2 \sigma^{2}}} d x=\frac{2}{\sigma \sqrt{2 \pi}} \int_{0}^{+\infty} x e^{-\frac{x^{2}}{2 \sigma^{2}}} d x \\
& =\frac{2}{\sigma \sqrt{2 \pi}}\left[-\sigma^{2} e^{-\frac{x^{2}}{2 \sigma^{2}}}\right]_{0}^{+\infty}=\sigma \sqrt{\frac{2}{\pi}}
\end{aligned}
$$

Lemma 5 Let $Z=(X, Y)^{T}$ be a Gaussian random vector with zero mean and covariance matrix

$\mathbb{E}\left(Z Z^{T}\right)=\left(\begin{array}{cc}a^{2} & a b \sin \theta \\ a b \sin \theta & b^{2}\end{array}\right)$,

with $\theta \in\left[-\frac{\pi}{2}, \frac{\pi}{2}\right]$. Then, one has

$\mathbb{E}(|X Y|)=\frac{2|a b|}{\pi}(\cos \theta+\theta \sin \theta)$.

Proof If $a=0$ or $b=0$, then $\mathbb{E}(X Y)=0$ so there is nothing more to prove. Hence we can assume that $a b \neq 0$ and set $X^{\prime}=X / a, Y^{\prime}=Y / b$, so that

$\mathbb{E}|X Y|=|a b| \cdot \mathbb{E}\left|X^{\prime} Y^{\prime}\right|$,

where the covariance of $Z^{\prime}=\left(X^{\prime}, Y^{\prime}\right)^{T}$ is

$C=\mathbb{E}\left(Z^{\prime} Z^{\prime T}\right)=\left(\begin{array}{cc}1 & \sin \theta \\ \sin \theta & 1\end{array}\right)$
If $|\sin \theta|=1$, then $Y^{\prime}=X^{\prime} \sin \theta$ almost surely, so that $\mathbb{E}\left|X^{\prime} Y^{\prime}\right|=\mathbb{E} X^{\prime 2}=1$ and $\mathbb{E}|X Y|=|a b|$ by (44). Hence, we assume in the following that $|\theta|<\frac{\pi}{2}$. Now we have

$C^{-1}=\frac{1}{\cos ^{2} \theta}\left(\begin{array}{cc}1 & -\sin \theta \\ -\sin \theta & 1\end{array}\right)$,

so that $\mathbb{E}\left|X^{\prime} Y^{\prime}\right|$ equals

$\frac{1}{2 \pi \cos \theta} \int_{\mathbb{R}^{2}}|x y| \exp \left(-\frac{x^{2}+y^{2}-2 x y \sin \theta}{2 \cos ^{2} \theta}\right) d x d y$.

Using symmetry considerations, this formula can be rewritten under the form

$\mathbb{E}\left|X^{\prime} Y^{\prime}\right|=\frac{I(\theta)+I(-\theta)}{\pi \cos \theta}$

with $I(\theta)=\int_{0}^{+\infty} \int_{0}^{+\infty} x y \exp \left(-\frac{x^{2}+y^{2}-2 x y \sin \theta}{2 \cos ^{2} \theta}\right) d x d y$.

Using polar coordinates, we then get

$$
\begin{aligned}
I(\theta)= & \int_{0}^{+\infty} \int_{0}^{\frac{\pi}{2}} r^{2} \cos \varphi \sin \varphi \\
& \quad \exp \left(-\frac{r^{2}}{2 \cos ^{2} \theta}(1-2 \cos \varphi \sin \varphi \sin \theta)\right) r d r d \varphi \\
= & \int_{0}^{\frac{\pi}{2}}\left(\cos \varphi \sin \varphi \int_{0}^{+\infty} r^{3} e^{-\alpha(\varphi) r^{2}} d r\right) d \varphi
\end{aligned}
$$

with $\alpha(\varphi)=\frac{1-2 \cos \varphi \sin \varphi \sin \theta}{2 \cos ^{2} \theta} \geq 0$.

Integrating by part the inside integral yields

$$
\begin{aligned}
& \int_{0}^{+\infty} r^{3} e^{-\alpha(\varphi) r^{2}} d r \\
& =\left[r^{2} \cdot \frac{1}{-2 \alpha(\varphi)} e^{-\alpha(\varphi) r^{2}}\right]_{0}^{+\infty}-\frac{1}{-2 \alpha(\varphi)} \int_{0}^{+\infty} 2 r e^{-\alpha(\varphi) r^{2}} d r \\
& =\frac{1}{2 \alpha(\varphi)^{2}} .
\end{aligned}
$$

Thus we have

$$
\begin{aligned}
I(\theta) & =\int_{0}^{\frac{\pi}{2}} \cos \varphi \sin \varphi \cdot \frac{\left(2 \cos ^{2} \theta\right)^{2}}{2(1-2 \cos \varphi \sin \varphi \sin \theta)^{2}} d \varphi \\
& =2 \cos ^{4} \theta \cdot \int_{0}^{\frac{\pi}{2}} \frac{d \varphi}{\left(\cos ^{-2} \varphi-2 \tan \varphi \sin \theta\right)^{2}} \frac{d \varphi}{\cos ^{2} \varphi} \\
& =2 \cos ^{4} \theta \cdot \int_{0}^{+\infty} \frac{t}{\left(1+t^{2}-2 t \sin \theta\right)^{2}} d t \quad(t=\tan \varphi) \\
& =2 \cos ^{4} \theta \cdot \int_{0}^{+\infty} \frac{t}{\left((t-\sin \theta)^{2}+\cos ^{2} \theta\right)^{2}} d t \\
& =2 \cos ^{4} \theta \cdot \int_{-\sin \theta}^{+\infty} \frac{u+\sin \theta}{\left(u^{2}+\cos ^{2} \theta\right)^{2}} d u \quad(u=t-\sin \theta) .
\end{aligned}
$$

Now usual integration formulae give (for $a>0$ ),

$\int \frac{u}{\left(u^{2}+a^{2}\right)^{2}} d u=\frac{-1}{2\left(u^{2}+a^{2}\right)}$

and $\int \frac{1}{\left(u^{2}+a^{2}\right)^{2}} d u=\frac{1}{2 a^{3}} \arctan \frac{u}{a}+\frac{u}{2 a^{2}\left(u^{2}+a^{2}\right)}$, 
so that $I(\theta)$ equals

$$
\begin{aligned}
& I(\theta)=2 \cos ^{4} \theta\left(\left[\frac{-1}{2\left(u^{2}+\cos ^{2} \theta\right)^{2}}\right]_{-\sin \theta}^{+\infty}\right. \\
& \left.+\sin \theta\left[\frac{1}{2 \cos ^{3} \theta} \arctan \frac{u}{\cos \theta}+\frac{u}{2 \cos ^{2} \theta\left(u^{2}+\cos ^{2} \theta\right)}\right]_{-\sin \theta}^{+\infty}\right) \\
& \quad=2 \cos ^{4} \theta\left(\frac{1}{2}+\sin \theta\left(\frac{\pi}{2 \cos ^{3} \theta}+\frac{\theta}{2 \cos ^{3} \theta}+\frac{\sin \theta}{2 \cos ^{2} \theta}\right)\right) \\
& \quad=\cos ^{4} \theta+\pi \sin \theta \cos \theta+\theta \sin \theta \cos \theta+\sin ^{2} \theta \cos ^{2} \theta \\
& \quad=\cos ^{2} \theta+\pi \sin \theta \cos \theta+\theta \sin \theta \cos \theta .
\end{aligned}
$$

Then, $I(\theta)+I(-\theta)=2 \cos \theta(\cos \theta+\theta \sin \theta)$ and we conclude by (44) and (45) that

$\mathbb{E}|X Y|=\frac{2|a b|}{\pi}(\cos \theta+\theta \sin \theta)$.

Proof of Theorem 1

Writing $U=u * W$, we have by linearity $\partial_{x} \dot{U}=\left(\partial_{x} \dot{u}\right) * W$,

so that the discrete random field $\partial_{x} \dot{U}$ is a stationary Gaussian field whose marginal distributions have zero mean and variance

$\mathbb{E}\left(\left(\partial_{x} \dot{U}(\mathbf{x})\right)^{2}\right)=\frac{1}{M N} \sum_{\mathbf{y} \in \Omega}\left(\partial_{x} \dot{u}(\mathbf{x}-\mathbf{y})\right)^{2}=\frac{\alpha_{x}^{2}}{M N}$.

From Lemma 4, we hence get that for any $\mathbf{x} \in \Omega$,

$\mathbb{E}\left(\left|\partial_{x} \dot{U}(\mathbf{x})\right|\right)=\frac{\alpha_{x}}{\sqrt{M N}} \sqrt{\frac{2}{\pi}}$,

and by using a similar reasoning on $\partial_{y} \dot{U}$, we obtain (10).

We now consider the variance of $\operatorname{TV}(U)$. We have

$$
\begin{aligned}
\mathbb{E}\left(\mathrm{TV}(U)^{2}\right)= & \sum_{\mathbf{x}, \mathbf{y} \in \Omega} \mathbb{E}\left|\partial_{x} \dot{U}(\mathbf{x}) \partial_{x} \dot{U}(\mathbf{y})\right|+\mathbb{E}\left|\partial_{x} \dot{U}(\mathbf{x}) \partial_{y} \dot{U}(\mathbf{y})\right| \\
& +\mathbb{E}\left|\partial_{y} \dot{U}(\mathbf{x}) \partial_{x} \dot{U}(\mathbf{y})\right|+\mathbb{E}\left|\partial_{y} \dot{U}(\mathbf{x}) \partial_{y} \dot{U}(\mathbf{y})\right|
\end{aligned}
$$

Writing $\mathbf{z}=\mathbf{y}-\mathbf{x}$ and using the stationarity of $\nabla \dot{U}$, the quantity $\mathbb{E}\left(\mathrm{TV}(U)^{2}\right)$ can be rewritten

$$
\begin{aligned}
M N \sum_{\mathbf{x} \in \Omega, \mathbf{y} \in \Omega} & \mathbb{E}\left|\partial_{x} \dot{U}(0) \partial_{x} \dot{U}(\mathbf{z})\right|+\mathbb{E}\left|\partial_{x} \dot{U}(0) \partial_{y} \dot{U}(\mathbf{z})\right| \\
& +\mathbb{E}\left|\partial_{y} \dot{U}(0) \partial_{x} \dot{U}(\mathbf{z})\right|+\mathbb{E}\left|\partial_{y} \dot{U}(0) \partial_{y} \dot{U}(\mathbf{z})\right|
\end{aligned}
$$

Each term of this sum can be written under the form $\mathbb{E}|X Y|$ where $(X, Y)$ is a zero-mean 2-dimensional Gaussian vector with covariance matrix

$\left(\begin{array}{ll}\mathbb{E}\left(X^{2}\right) & \mathbb{E}(X Y) \\ \mathbb{E}(X Y) & \mathbb{E}\left(Y^{2}\right)\end{array}\right)$

For the second term of (46) for example, we have $X=\partial_{x} \dot{U}(0)$ and $Y=\partial_{y} \dot{U}(\mathbf{z})$, thus

$$
\begin{aligned}
\mathbb{E}(X Y) & =\mathbb{E}\left(\sum_{\mathbf{x} \in \Omega, \mathbf{y} \in \Omega} \partial_{x} \dot{u}(-\mathbf{x}) \partial_{y} \dot{u}(\mathbf{z}-\mathbf{y}) W(\mathbf{x}) W(\mathbf{y})\right) \\
& =\frac{1}{M N} \sum_{\mathbf{x} \in \Omega} \partial_{x} \dot{u}(\mathbf{x}) \partial_{y} \dot{u}(\mathbf{z}+\mathbf{x})=\frac{1}{M N} \Gamma_{x y}(\mathbf{z})
\end{aligned}
$$

and the covariance matrix of $(X, Y)$ is

$$
\frac{1}{M N}\left(\begin{array}{cc}
\alpha_{x}^{2} & \Gamma_{x y}(\mathbf{z}) \\
\Gamma_{x y}(\mathbf{z}) & \alpha_{y}^{2}
\end{array}\right)
$$

so that thanks to Lemma 5 we obtain

$$
\mathbb{E}|X Y|=\frac{2 \alpha_{x} \alpha_{y}}{\pi M N} \cdot \widetilde{\omega}\left(\frac{\Gamma_{x y}(\mathbf{z})}{\alpha_{x} \alpha_{y}}\right),
$$

with $\widetilde{\omega}(t)=t \arcsin t+\sqrt{1-t^{2}}=\omega(t)+1$. Combining all terms arising from (46), we finally obtain that

$$
\begin{aligned}
\mathbb{E}\left(\operatorname{TV}(U)^{2}\right)=\frac{2}{\pi} \sum_{\mathbf{z} \in \Omega} & \alpha_{x}^{2} \widetilde{\omega}\left(\frac{\Gamma_{x x}(\mathbf{z})}{\alpha_{x}^{2}}\right) \\
& +2 \alpha_{x} \alpha_{y} \widetilde{\omega}\left(\frac{\Gamma_{x y}(\mathbf{z})}{\alpha_{x} \alpha_{y}}\right)+\alpha_{y}^{2} \widetilde{\omega}\left(\frac{\Gamma_{y y}(\mathbf{z})}{\alpha_{y}^{2}}\right)
\end{aligned}
$$

and the announced result follows from

$\operatorname{Var}(\mathrm{TV}(U))=\mathbb{E}\left(\operatorname{TV}(U)^{2}\right)-(\mathbb{E}(\operatorname{TV}(U)))^{2}$,

which simply amounts to change $\widetilde{\omega}$ into $\omega$ in (47).

\section{Unimodal regression}

In this appendix, we detail an algorithm to compute the distance from a signal $s=(s(1), s(2), \ldots, s(n)) \in \mathbb{R}^{n}$ to the set $U$ of unimodal signals of size $n$, defined by

$U=\bigcup_{1 \leq i \leq n} C_{i} \cap D_{i}$

where $C_{i}=\left\{p \in \mathbb{R}^{n}, p(1) \leq p(2) \leq \ldots \leq p(i)\right\}$

and $D_{i}=\left\{p \in \mathbb{R}^{n}, p(i) \geq p(i+1) \geq \ldots \geq p(n)\right\}$

(with the natural convention $C_{1}=D_{n}=\mathbb{R}^{n}$ ). The algorithm we use is due to Frisen [15]. It is based on the fact that $U$ can also be written

$$
U=\bigcup_{1 \leq i \leq n-1} C_{i} \cap D_{i+1}
$$

which entails $d(s, U)=\min _{1 \leq i \leq n-1} d_{i}$ with

$$
\begin{aligned}
d_{i}^{2} & =\min _{p \in C_{i} \cap D_{i+1}}\|p-s\|_{2}^{2} \\
& =\min _{p \in C_{i}} \sum_{k=1}^{i}(p(k)-s(k))^{2}+\min _{q \in D_{i+1}} \sum_{k=i+1}^{n}(q(k)-s(k))^{2} .
\end{aligned}
$$

These two monotone regression problems are independent, and can be solved in time $\mathcal{O}(n)$ using the simple Pool Adjacent Violators algorithm described in [1] (see Algorithm 4). Thus, the computation of $d(s, U)$ can be realized in time $\mathcal{O}\left(n^{2}\right)$ (Algorithm 5). Note that in fact the unimodal regression problem can be solved in time $\mathcal{O}(n)$ with a more sophisticated algorithm (see [34]), but considering the small value of $n$ we use in Section $5.3(n=20)$, the gain obtained with this algorithm would be negligible compared to other steps (e.g., Fourier transforms) of the deblurring process. 

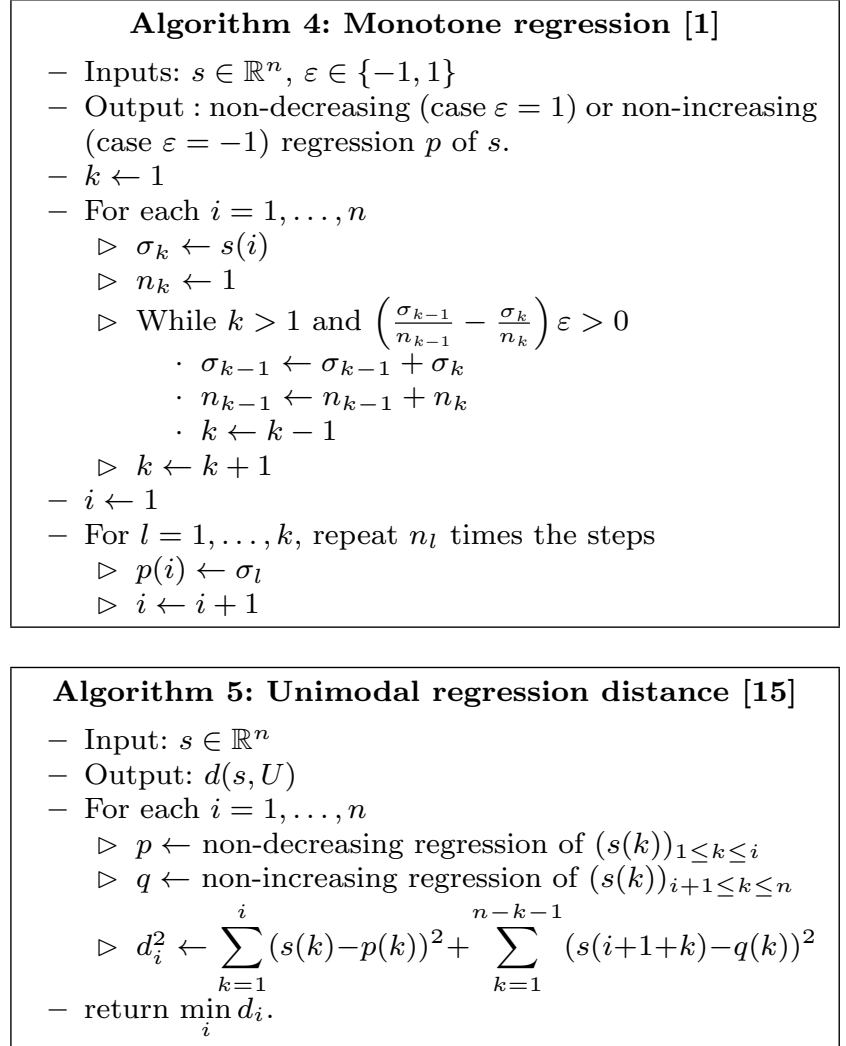

\section{E Oracle deconvolution filter}

Consider a blurry and noisy image $v=\kappa * u_{0}+n$, obtained from an image $u_{0}$ after a convolution by a kernel $\kappa$ and the addition of a Gaussian white noise $n$ with standard deviation $\sigma^{2}$. In this appendix, we show how to compute the oracle kernel $k_{0}$ which provides, in average with respect to $n$, the best linear estimate of $u_{0}$ that can be computed from $v$. This oracle kernel is defined by

$k_{0}=\arg \min _{k} \mathbb{E}\left(\left\|u_{0}-k *\left(\kappa * u_{0}+W\right)\right\|_{2}^{2}\right)$,

where $W$ is a Gaussian white noise with variance $\sigma^{2}$. The arg min can be taken over various kernel spaces, here we consider the set of kernels obtained by rotating a radial linearly interpolated profile, that is

$\forall \boldsymbol{\xi} \in \Omega, \quad \hat{k}(\boldsymbol{\xi})=r(\lfloor|\boldsymbol{\xi}|\rfloor)(\lceil|\boldsymbol{\xi}|\rceil-|\boldsymbol{\xi}|)+r(\lceil|\boldsymbol{\xi}|\rceil)(|\boldsymbol{\xi}|-\lfloor|\boldsymbol{\xi}|\rfloor)$, where $(r(0), \ldots, r(d-1)) \in \mathbb{R}^{d}$,

$|\boldsymbol{\xi}|^{2}=2(d-1)^{2}\left(\frac{\xi_{1}^{2}}{M^{2}}+\frac{\xi_{2}^{2}}{N^{2}}\right)=\frac{2(d-1)^{2}}{4 \pi^{2}}\|\boldsymbol{\xi}\|^{2}$,

and $\lfloor t\rfloor$ and $\lceil t\rceil$ denote respectively the lower and upper integer part of $t \in \mathbb{R}$ (we also set $\hat{k}(\boldsymbol{\xi})=0$ when $|\boldsymbol{\xi}|>d-1$ ). This interpolation formula naturally involves the disjoint subsets

$\widehat{\Omega}_{l}=\{\boldsymbol{\xi} \in \Omega, l \leq|\boldsymbol{\xi}|<l+1\}$.

Since $W$ is a white Gaussian noise, the cost function of (48) can be written

$$
\begin{aligned}
& \left\|u_{0}-k * \kappa * u_{0}\right\|_{2}^{2}+\sigma^{2} M N\|k\|_{2}^{2} \\
= & \frac{1}{M N} \sum_{\boldsymbol{\xi} \in \Omega}\left|\widehat{u_{0}}(\boldsymbol{\xi})\right|^{2}|1-\hat{k}(\boldsymbol{\xi}) \hat{\kappa}(\boldsymbol{\xi})|^{2}+\sigma^{2} M N|\hat{k}(\boldsymbol{\xi})|^{2},
\end{aligned}
$$

which, when $\hat{k}$ is radial and when $\kappa$ is supposed to be symmetrical, transforms into

$$
\begin{array}{r}
\frac{1}{M N} \sum_{l=0}^{d-1} \sum_{\boldsymbol{\xi} \in \widehat{\Omega}_{l}}\left|\widehat{u_{0}}(\boldsymbol{\xi})\right|^{2}(1-\kappa(\boldsymbol{\xi}) r(l)(l+1-|\boldsymbol{\xi}|) \\
-\kappa(\boldsymbol{\xi}) r(l+1)(|\boldsymbol{\xi}|-l))^{2} \\
+\sigma^{2} M N(r(l)(l+1-|\boldsymbol{\xi}|)+r(l+1)(|\boldsymbol{\xi}|-l))^{2} .
\end{array}
$$

This is a quadratic function in $r$, and its unique minimum is characterized by the vanishing-gradient condition, which can be written $A r=b$, where $A=\left(\left(a_{k, l}\right)\right)_{0 \leq k, l \leq d-1}$ and $b=\left(b_{l}\right)_{0 \leq l \leq d-1}$ are defined by

$$
\begin{aligned}
a_{l, l} & =\sum_{\boldsymbol{\xi} \in \widehat{\Omega}_{l}}(l+1-|\boldsymbol{\xi}|)^{2}\left(|\kappa(\boldsymbol{\xi})|^{2}\left|\widehat{u_{0}}(\boldsymbol{\xi})\right|^{2}+\sigma^{2} M N\right) \\
& +\sum_{\boldsymbol{\xi} \in \widehat{\Omega}_{l-1}}(|\boldsymbol{\xi}|-l+1)^{2}\left(|\kappa(\boldsymbol{\xi})|^{2}\left|\widehat{u_{0}}(\boldsymbol{\xi})\right|^{2}+\sigma^{2} M N\right) \\
a_{l, l+1} & =\sum_{\boldsymbol{\xi} \in \widehat{\Omega}_{l}}(l+1-|\boldsymbol{\xi}|)(|\boldsymbol{\xi}|-l)\left(|\kappa(\boldsymbol{\xi})|^{2}\left|\widehat{u_{0}}(\boldsymbol{\xi})\right|^{2}+\sigma^{2} M N\right) \\
a_{l, l-1} & =\sum_{\boldsymbol{\xi} \in \widehat{\Omega}_{l-1}}(|\boldsymbol{\xi}|-l+1)(l-|\boldsymbol{\xi}|)\left(|\kappa(\boldsymbol{\xi})|^{2}\left|\widehat{u_{0}}(\boldsymbol{\xi})\right|^{2}+\sigma^{2} M N\right) \\
a_{l, m}= & 0 \text { for }|l-m|>1 \\
b_{l} & =\sum_{\boldsymbol{\xi} \in \widehat{\Omega}_{l}}(t+1-|\boldsymbol{\xi}|)^{2}\left(|\kappa(\boldsymbol{\xi})|\left|\widehat{u_{0}}(\boldsymbol{\xi})\right|^{2}\right) \\
& +\sum_{\boldsymbol{\xi} \in \widehat{\Omega}_{l-1}}(|\boldsymbol{\xi}|-l+1)^{2}\left(|\kappa(\boldsymbol{\xi})|\left|\widehat{u_{0}}(\boldsymbol{\xi})\right|^{2}\right) .
\end{aligned}
$$

This linear system associated to the tridiagonal matrix $A$ can be solved with standard numerical techniques. The solution is the oracle radial profile $r_{0}$, from which the DFT of the oracle kernel $k_{0}$ can be defined by

$\forall l, \forall \boldsymbol{\xi} \in \widehat{\Omega}_{l}, \quad \widehat{k_{0}}(\boldsymbol{\xi})=r_{0}(l)(l+1-|\boldsymbol{\xi}|)+r_{0}(l+1)(|\boldsymbol{\xi}|-l)$.

Remark: One can also consider the minimization problem (48) on the set of all kernels $k$. It is easy to deduce from (50) that the corresponding oracle kernel is given in Fourier domain by

$\forall \boldsymbol{\xi} \in \Omega, \quad \hat{k}(\boldsymbol{\xi})=\frac{\hat{\kappa}(\boldsymbol{\xi})^{*}\left|\widehat{u_{0}}(\boldsymbol{\xi})\right|^{2}}{|\kappa(\boldsymbol{\xi})|^{2}\left|\widehat{u_{0}}(\boldsymbol{\xi})\right|^{2}+\sigma^{2} M N}$

One can notice that, making the assumption $|\hat{u}(\boldsymbol{\xi})|^{2}=c\|\boldsymbol{\xi}\|^{-2}$ (see the discussion at the end of Appendix A), and setting $\lambda=\sigma^{2} M N / c$, the corresponding filter is exactly the one that optimizes the criterion (26).

\section{References}

1. Ayer, M., Brunk, H. D., Ewing, G. M., Reid, W. T., Silverman, E., "An empirical distribution function for sampling with incomplete information", The annals of mathematical statistics, pp. 641-647, 1955.

2. Blanchet, G., Moisan, L., Rougé, B., "A linear prefilter for image sampling with ringing artifact control", Proceedings of Int. Conf. on Image Processing (ICIP), vol 3 , pp. $577-580,2005$. 
3. Blanchet, G., Moisan, L., Rougé, B., "Measuring the Global Phase Coherence of an Image", Proceedings of Int. Conf. on Image Processing (ICIP), pp. 1176-1179, 2008.

4. Blanchet, G., Moisan, L., "An Explicit Sharpness Index Related to Global Phase Coherence", Proceedings of Int. Conf. on Acoustics, Speech, and Signal Processing (ICASSP), pp. 1065-1068, 2012.

5. Calderero, F., Moreno, P., "Evaluation of Sharpness Measures and Proposal of a Stop Criterion for Reverse Diffusion in the Context of Image Deblurring," Proceedings of Int. Conf. on Computer Vision Theory and Applications (VISAPP), 2013.

6. Chambolle, A., "An algorithm for total variation minimization and applications", Journal of Mathematical Imaging and Vision, vol. 20(1-2), pp. 89-97, 2004.

7. Chandler, D.M., "Seven Challenges in Image Quality Assessment: Past, Present, and Future Research", ISRN Signal Processing, article id. 905685, 2013.

8. Deriugin, N.G., "The power spectrum and the correlation function of the television signal", Telecommunications 1(7), pp. 1-12, 1956.

9. Desolneux, A., Moisan, L., Morel, J.-M., "Dequantizing image orientation", IEEE Transactions on Image Processing, vol. 11(10), pp. 1129-1140, 2002.

10. Desolneux, A., Moisan, L., Morel, J.-M., From Gestalt Theory to Image Analysis: A Probabilistic Approach, Springer-Verlag, collection "Interdisciplinary Applied Mathematics", vol. 34, 2008.

11. Desolneux, A., Moisan, L., Ronsin, S., "A Compact Representation of Random Phase and Gaussian Textures", Proceedings of Int. Conf. on Acoustics, Speech, and Signal Processing (ICASSP), pp. 1381-1384, 2012.

12. Ekeland, I., Temam, R., Convex Analysis and Variational Problems, SIAM, Classics in Applied Mathematics, 1999.

13. Ferzli, R., Karam, L.J., "A No-reference Objective Image Sharpness Metric Based on the Notion of Just Noticeable Blur (JNB)", IEEE Transactions on Image Processing, vol 18(4), pp. 717-728, 2009.

14. Grosjean, B., Moisan, L., "A-contrario detectability of spots in textured backgrounds", Journal of Mathematical Imaging and Vision, vol. 33(3), pp.313-337, 2009.

15. Frisen, M., "Unimodal Regression", The Statistician, vol. 35(4), pp. 479-485, 1986.

16. Galerne, B., Gousseau, Y., Morel, J.-M., "Random Phase Textures : Theory and Synthesis", IEEE Transactions on Image Processing, vol. 20(1), pp. 257-267, 2011.

17. Hassen, R., Wang, Z., Salama, M., "No-Reference Image Sharpness Assessment Based on Local Phase Coherence Measurement", Proceedings of Int. Conf. on Acoustics, Speech, and Signal Processing (ICASSP), pp. 2434-2437, 2010.

18. Janson., S., "Normal convergence by higher semiinvariants with applications to sums of dependent random variables and random graphs", The Annals of Probability, vol. 16(1), pp. 305-312, 1988.

19. Kovesi, P., "Phase Congruency: a Low-level Image Invariant", Psychological Research, vol. 64, pp. 136-148, 2000.

20. Kovesi, P., "Image features from phase congruency", Technical Report, 1999.

21. Louchet, C., Moisan, L., "Total Variation denoising using iterated conditional expectation", Proceedings of European Signal Processing Conference (EUSIPCO), 2014.

22. Leclaire, A., Moisan, L., "Blind Deblurring Using a Simplified Sharpness Index", Proceedings of Int. Conf. on Scale Space and Variational Methods in Computer Vision (SSVM), pp. 86-97, 2013.
23. Leclaire, A., Moisan, L., "Une Variante Non Périodique du Sharpness Index", Actes du GRETSI (in french), pp. 86-97, 2013.

24. Levin, A., Weiss, Y., Durand, F., Freeman, W.T., "Efficient Marginal Likelihood Optimization in Blind Deconvolution", Proc. of IEEE Conf. on Computer Vision and Pattern Recognition (CVPR), pp. 2657-2664, 2011.

25. Liu, Y., Wang, J., Cho, S., Finkelstein, A., Rusinkiewicz, S., "A No-Reference Metric for Evaluating the Quality of Motion Deblurring", ACM Transactions on Graphics (TOG), vol. 32(6), pp. 175, 2013.

26. Marziliano, P.,Dufaux, F., Winkler, S., Ebrahimi, T., "Perceptual blur and ringing metrics: Application to JPEG2000", Signal Processing: Image Communication, vol. 19(2), pp. 163-172, 2004.

27. Moisan, L., "How to discretize the Total Variation of an image?", Proc. Appl. Math. Mech., vol. 7, pp. 10419071041908, 2007.

28. Moisan, L., "Periodic Plus Smooth Image Decomposition", Journal of Mathematical Imaging and Vision, vol. 39(2), pp. 120-145, 2011.

29. Morrone, M.C., Burr, D.C., "Feature Detection in Human Vision: a Phase-Dependent Energy Model", Proc. of the Royal Society of London, Series B, pp. 221-245, 1988.

30. Oppenheim, A.V., Lim, J.S., "The Importance of Phase in Signals", Proceedings of the IEEE, vol. 69, pp. 529$541,1981$.

31. Ruderman, D. L., "The statistics of natural images", Network: computation in neural systems 5(4), pp. 517-548, 1994.

32. Rudin, L.I., Osher, S., Fatemi, E., Nonlinear Total Variation Based Noise Removal Algorithms, Physica D, vol. 60, pp. 259-268, 1992.

33. Shevtsova., I., "An improvement of convergence rate estimates in the Lyapunov theorem", Doklady Mathematics, vol. 82, pp. 862-864, 2010.

34. Stout, Q.F., "Unimodal regression via prefix isotonic regression", Computational Statistics and Data Analysis, vol. 53, pp. 289-297, 2008.

35. Takeda, H., Farsiu, S., and Milanfar, P., "Kernel regression for image processing and reconstruction", IEEE Transactions on Image Processing, vol.16(2), pp. 349366, 2007.

36. van Wijk, J.J., "Spot noise texture synthesis for data visualization", ACM SIGGRAPH Computer Graphics, vol. 25(4), pp. 309-318, 1991.

37. Vu, C.T., Chandler, D.M., "S3: a spectral and spatial sharpness measure", Advances in Multimedia, Proc. of Int. Conf. MMEDIA, pp. 37-43, 2009.

38. Wang, Z., Bovik, A., Modern Image Quality Assessment (Synthesis Lectures on Image, Video, and Multimedia Processing), Morgan \& Claypool Publishers, 2006.

39. Wang, Z., Simoncelli, E.P., "Local Phase Coherence and the Perception of Blur", Advances in Neural Information Processing Systems (NIPS), vol. 16, pp. 786-792, 2004.

40. Zhu, X., Milanfar, P., "Automatic Parameter Selection for Denoising Algorithms Using a No-Reference Measure of Image Content", IEEE Transactions on Image Processing, vol. 19(12), pp. 3116-3132, 2010. 